The Annals of Applied Statistics

\title{
ASYMMETRIC CONDITIONAL CORRELATIONS IN STOCK RETURNS
}

\author{
By Hui Jiang ${ }^{1}$, Patrick W. SaART ${ }^{2}$ And YingCun XiA ${ }^{1,3}$ \\ National University of Singapore, Singapore ${ }^{1}$ \\ Newcastle University, United Kingdom ${ }^{2}$ \\ University of Electronic Science and Technology, China ${ }^{3}$
}

\section{Supplementary Material}

This appendix provides detailed technical proofs for theorems in the main text, and also the imposed assumptions. In addition, some necessary lemmas and the proofs are presented accordingly.

Appendix A Theoretical justification. To make statistical inference, we need to find the asymptotic distribution of the estimators, including those for $\mu_{k}(u), \sigma_{k}^{2}(u), \varrho_{k, \ell}(u)$, $F_{j}(u)$ and $a_{k \ell}, b_{k \ell}^{[j]}, k=1, \ldots, m, 1 \leq k<\ell \leq m, j=1, \ldots, p$. The assumptions needed for our analysis are listed below.

(C1) Let $f_{U}(\cdot)$ denote the marginal density of $U_{t}$, and $f_{s}(\cdot, \cdot)$ denote the joint density of $\left(U_{t}, U_{t+s}\right)$. Suppose that $f(\cdot)$ has a bounded support, such as $[c, d], f_{U}(u)>0$, and $\left|f_{U}(u)-f_{U}\left(u^{\prime}\right)\right| \leq \Delta_{1}\left|u-u^{\prime}\right|$ for all given points $u, u^{\prime} \in[c, d]$ and some $\Delta_{1}>0$. Meanwhile, $f_{s}\left(u_{0}, u_{s}\right)>0$ for $u_{0}, u_{s} \in[c, d]$. Further, $\sup _{u \in[c, d]} f_{U}(u) \leq L_{0}<\infty$, $\sup _{u_{0}, u_{s} \in[c, d]} f_{s}\left(u_{0}, u_{s}\right) \leq L_{1}<\infty$.

(C2) $E\left|r_{k, t}\right|^{4(1+\delta)} \leq L_{2}<\infty, E\left|\epsilon_{k, \ell, t}\right|^{4(1+\delta)} \leq L_{2}<\infty$, for $k, \ell=1, \ldots, m, t=1, \ldots, n$, and some $\delta>0$. Meanwhile,

$$
\begin{aligned}
& \sup _{u_{0} \in[c, d]} E\left[\left|r_{k, t}\right|^{4(1+\delta)} \mid U_{t}=u_{0}\right] \leq L_{2}<\infty, \\
& \sup _{u_{0} \in[c, d]} E\left[\left|\epsilon_{k, \ell, t}\right|^{4(1+\delta)} \mid U_{t}=u_{0}\right] \leq L_{2}<\infty,
\end{aligned}
$$




$$
\begin{aligned}
& \sup _{u_{0}, u_{s} \in[c, d]} E\left[\left|\epsilon_{k, \ell, t}\right| \mid U_{t}=u_{0}, U_{t+s}=u_{s}\right] \leq L_{2}<\infty, \\
& \sup _{u_{0}, u_{s} \in[c, d]} E\left[\mid \epsilon_{k, \ell, t} \epsilon_{k, \ell, t+s} \| U_{t}=u_{0}, U_{t+s}=u_{s}\right] \leq L_{2}<\infty,
\end{aligned}
$$

for all $s \in \mathbb{Z}$ and some sufficiently large $L_{2}$. Moreover, for particular $k_{1}, k_{2}$ and $\ell_{1}, \ell_{2}$,

$$
\begin{aligned}
& E\left\{\epsilon_{k_{1}, \ell_{1}, t} \epsilon_{k_{2}, \ell_{2}, t} \mid U_{t}=u_{0}\right\}=0, \quad \text { if }\left\{k_{1}, \ell_{1}\right\} \neq\left\{k_{2}, \ell_{2}\right\} \\
& E\left\{\epsilon_{k_{1}, \ell_{1}, t} \epsilon_{k_{2}, \ell_{2}, t+s} \mid U_{t}=u_{0}, U_{t+s}=u_{s}\right\}=0, \quad \text { if }\left\{k_{1}, \ell_{1}\right\} \neq\left\{k_{2}, \ell_{2}\right\} .
\end{aligned}
$$

(C3) The time series $\left\{\left(r_{1, t}, r_{2, t}, \cdots, r_{m, t}, U_{t}\right): t=1, \ldots, n\right\}$ are strictly stationary and strong mixing with mixing coefficient $\alpha(N) \leq C N^{-\beta}$ for some $C>0$ and $\beta>2+\frac{2}{\delta}$ for the same $\delta$ as in (C2). Furthermore, suppose that $\left(r_{1, t}, r_{2, t}, \cdots, r_{m, t}, U_{t}\right)$ has the same distribution with $\left(r_{1}, r_{2}, \cdots, r_{m}, U\right)$.

(C4) (i) $\mu_{k}(u), \sigma_{k}^{2}(u), k=1, \ldots, m$ are differentiable, and $\mu_{k}^{\prime \prime}(u), \sigma_{k}^{2^{\prime \prime}}(u)$ are uniformly continuous.

(ii) $F_{j}(\cdot), j=1, \ldots, p$ are differentiable, and $F_{j}^{\prime \prime}(\cdot), j=1, \ldots, p$ are uniformly continuous. In addition, the coefficients $a_{k \ell}, b_{k \ell}^{[j]}$ are bounded by some constants $\bar{a}$, $\bar{b}<\infty$, i.e. $\left|a_{k \ell}\right|<\bar{a},\left|b_{k \ell}^{[j]}\right| \leq \bar{b}$ for all $1 \leq k<\ell \leq m$ and $j=1, \ldots, p$.

(C5) The continuous symmetric kernel function $K(\cdot)$ has the following properties:

(i) $\int|K(v)| d v<\infty, \int K^{2}(v) d v<\infty$, and $\int K(v) d v=1, \int v K(v) d v=0, \int v^{2} K(v) d v=$ $w_{2}^{K}, \int K^{2}(v) d v=\nu_{K}^{2}$.

(ii) For some $0<C_{1}<\infty$ and $0<\Delta_{2}<\infty$, either $K(\cdot)$ is a bounded function with a bounded support on $\mathbb{R}$ (such as $\left[-C_{1}, C_{1}\right]$ ), satisfying the Lipschitz condition, i.e. $\left|K\left(v_{1}\right)-K\left(v_{2}\right)\right| \leq \Delta_{2}\left|v_{1}-v_{2}\right|$, or $K(\cdot)$ is differentiable, when $v \rightarrow \infty$, $K(v) e^{c_{0} v} \rightarrow 0\left(c_{0}>0\right)$.

(iii) Let $K * K(v)=\int K(x) K(x+v) d x$, and $\nu_{K, K * K}=\int K(v) K * K(v) d v, \nu_{K * K}^{2}=$ $\int(K * K(v))^{2} d v$.

(C6) As $n \rightarrow \infty, h \rightarrow 0$, such that $h=O\left(n^{-\frac{1}{5}}\right)$.

At the beginning, we introduce the following lemma, which will serve as essential tools to derive asymptotic results for the estimators. The proof could be found in Fan (1996), Fan and Yao (2003) and Hansen (2008). 
Lemma A.1. Under the regularity conditions, for model $Y_{t}=m\left(U_{t}\right)+\sigma\left(U_{t}\right) \varepsilon_{t}, t=$ $1, \ldots, n$, where $\left(U_{t}, Y_{t}\right)$ is a strictly stationary time series, and $E\left\{\varepsilon_{t} \mid U_{t}\right\}=0$. Let $\hat{m}(u)$ be the local linear estimator of $m(u)$.

(i) We have uniformly

(A.1) $\hat{m}(u)=m(u)+\frac{1}{2} w_{2}^{K} m^{\prime \prime}(u) h^{2}+\frac{1}{n f_{U}(u)} \sum_{t=1}^{n} K_{h}\left(U_{t}-u\right) \sigma\left(U_{t}\right) \varepsilon_{t}+\delta_{n}$, where $\delta_{n}=o_{P}\left(h^{2}+\{\log n /(n h)\}^{1 / 2}\right)$.

(ii)

$$
\begin{array}{r}
\text { (A.2) } \sup _{u \in[c, d]}\left|\frac{1}{n} \sum_{t=1}^{n}\left[K_{h}\left(U_{t}-u\right) Y_{t}-E\left\{K_{h}\left(U_{t}-u\right) Y_{t}\right\}\right]\right|=O_{p}\left(\{\log n /(n h)\}^{1 / 2}\right), \\
(\mathrm{A} .3) \sup _{\substack{u, v \in[c, d] \\
n}}\left|\frac{1}{n} \sum_{t=1}^{n}\left[K_{h}\left(U_{t}-u\right) K_{h}\left(U_{t}-v\right) Y_{t}-E\left\{K_{h}\left(U_{t}-u\right) K_{h}\left(U_{t}-v\right) Y_{t}\right\}\right]\right| \\
=O_{p}\left(\frac{1}{h}\{\log n /(n h)\}^{1 / 2}\right),
\end{array}
$$

Denote $K_{h}\left(U_{t}-u\right)$ by $K_{h, t}(u)$, and denote $K * K_{h}\left(U_{t}-u\right)$ by $K * K_{h, t}(u)$. By this Lemma, we have the following results.

(a) Estimator of $\mu_{k}(u)$

$$
\hat{\mu}_{k}(u)-\mu_{k}(u)=\frac{1}{2} w_{2}^{K} \mu_{k}^{\prime \prime}(u) h^{2}+N_{1}(u)+\delta_{n},
$$

where

$$
N_{1}(u)=\frac{1}{n f_{U}(u)} \sum_{t=1}^{n} K_{h, t}(u) \sigma_{k}\left(U_{t}\right) \varepsilon_{k, t} \stackrel{d}{\rightarrow} N\left\{0,\left(n h f_{U}(u)\right)^{-1} \nu_{K}^{2} \sigma_{k}^{2}(u)\right\} .
$$

(b) Estimator of $\sigma_{k}^{2}(u)$.

$$
\hat{\sigma}_{k}^{2}(u)=\sigma_{k}^{2}(u)+\frac{1}{2} w_{2}^{K}\left(\sigma_{k}^{2}(u)\right)^{\prime \prime} h^{2}+N_{4}(u)+\delta_{n},
$$

where

$$
N_{2}(u)=\frac{1}{n f_{U}(u)} \sum_{t=1}^{n} K_{h, t}(u) \sigma_{k}^{2}\left(U_{t}\right) \xi_{k, t} \stackrel{d}{\rightarrow} N\left\{0, \frac{\nu_{K}^{2} \sigma_{k}^{4}(u) \sigma_{k}^{* 2}(u)}{n h f_{U}(u)}\right\},
$$

where $\xi_{k, t}=\varepsilon_{k, t}^{2}-1$ and $\sigma_{k}^{* 2}(u)=E\left(\xi_{k}^{2} \mid U=u\right)$. 
(c) Estimator of $\varrho_{k, \ell}(u): \hat{\varrho}_{k, \ell}(u)$. By the definition of $\varrho_{k, \ell}^{*}(u)$ and (A.1),

$$
\varrho_{k, \ell}^{*}(u)=\varrho_{k, \ell}(u)+\frac{1}{2} w_{2}^{K} \varrho_{k, \ell}^{\prime \prime}(u) h^{2}+\frac{1}{n f_{U}(u)} \sum_{t=1}^{n} K_{h, t}(u) \epsilon_{k, \ell, t}+\delta_{n} .
$$

From the definition of $\hat{\varrho}_{k, \ell}(u)$,

$$
\hat{\varrho}_{k, \ell}(u)=\varrho_{k, \ell}^{*}(u)+\frac{\sum_{t=1}^{n} W_{n, h}\left(U_{t}-u\right)\left(\hat{\varepsilon}_{k, t} \hat{\varepsilon}_{\ell, t}-\varepsilon_{k, t} \varepsilon_{\ell, t}\right)}{\sum_{t=1}^{n} W_{n, h}\left(U_{t}-u\right)} .
$$

Together with above results,

$$
\begin{gathered}
r_{k, t}-\hat{\mu}_{k}\left(U_{t}\right)=\sigma_{k}\left(U_{t}\right) \varepsilon_{k, t}-\frac{1}{2} \mu_{k}^{\prime \prime}\left(U_{t}\right) w_{2}^{K} h^{2}-\frac{1}{n f_{U}\left(U_{t}\right)} \sum_{q=1}^{n} K_{h, q}\left(U_{t}\right) \sigma_{k}\left(U_{q}\right) \varepsilon_{k, q}+\delta_{n}, \\
\frac{1}{\hat{\sigma}_{k}\left(U_{t}\right)}=\frac{1}{\sigma_{k}\left(U_{t}\right)}\left[1-\frac{\sigma_{k}^{2 \prime \prime}\left(U_{t}\right) w_{2}^{K} h^{2}}{4 \sigma_{k}^{2}\left(U_{t}\right)}-\frac{1}{2 n f_{U}\left(U_{t}\right) \sigma_{k}^{2}\left(U_{t}\right)} \sum_{q=1}^{n} K_{h, q}\left(U_{t}\right) \sigma_{k}^{2}\left(U_{q}\right) \xi_{k, q}+\delta_{n}\right],
\end{gathered}
$$

hence,

$$
\begin{aligned}
\hat{\varepsilon}_{k, t}= & \varepsilon_{k, t}-\frac{1}{2} w_{2}^{K}\left[\frac{\mu_{k}^{\prime \prime}\left(U_{t}\right)}{\sigma_{k}\left(U_{t}\right)}+\frac{\varepsilon_{k, t} \sigma_{k}^{2 \prime}\left(U_{t}\right)}{2 \sigma_{k}^{2}\left(U_{t}\right)}\right] h^{2}-\frac{1}{n \sigma_{k}\left(U_{t}\right) f_{U}\left(U_{t}\right)} \sum_{q=1}^{n} K_{h, q}\left(U_{t}\right) \sigma_{k}\left(U_{q}\right) \varepsilon_{k, q} \\
& -\frac{\varepsilon_{k, t}}{2 n f_{U}\left(U_{t}\right) \sigma_{k}^{2}\left(U_{t}\right)} \sum_{q=1}^{n} K_{h, q}\left(U_{t}\right) \sigma_{k}^{2}\left(U_{t}\right) \xi_{k, q}+\delta_{n},
\end{aligned}
$$

similarly,

$$
\begin{aligned}
\hat{\varepsilon}_{\ell, t}= & \varepsilon_{\ell, t}-\frac{1}{2} w_{2}^{K}\left[\frac{\mu_{\ell}^{\prime \prime}\left(U_{t}\right)}{\sigma_{\ell}\left(U_{t}\right)}+\frac{\varepsilon_{\ell, t} \sigma_{\ell}^{2 \prime}\left(U_{t}\right)}{2 \sigma_{\ell}^{2}\left(U_{t}\right)}\right] h^{2}-\frac{1}{n \sigma_{\ell}\left(U_{t}\right) f_{U}\left(U_{t}\right)} \sum_{q=1}^{n} K_{h, q}\left(U_{t}\right) \sigma_{\ell}\left(U_{q}\right) \varepsilon_{\ell, q} \\
& -\frac{\varepsilon_{\ell, t}}{2 n f_{U}\left(U_{t}\right) \sigma_{\ell}^{2}\left(U_{t}\right)} \sum_{q=1}^{n} K_{h, q}\left(U_{t}\right) \sigma_{\ell}^{2}\left(U_{t}\right) \xi_{\ell, q}+\delta_{n},
\end{aligned}
$$

thus,

$$
\begin{aligned}
\hat{\varepsilon}_{k, t} \hat{\varepsilon}_{\ell, t}-\varepsilon_{k, t} \varepsilon_{\ell, t}= & -\frac{1}{2} w_{2}^{K}\left[\frac{\mu_{k}^{\prime \prime}\left(U_{t}\right) \varepsilon_{\ell, t}}{\sigma_{k}\left(U_{t}\right)}+\frac{\mu_{\ell}^{\prime \prime}\left(U_{t}\right) \varepsilon_{k, t}}{\sigma_{\ell}\left(U_{t}\right)}+\left(\frac{\sigma_{k}^{2^{\prime \prime}}\left(U_{t}\right)}{2 \sigma_{k}^{2}\left(U_{t}\right)}+\frac{\sigma_{\ell}^{2^{\prime \prime}}\left(U_{t}\right)}{2 \sigma_{\ell}^{2}\left(U_{t}\right)}\right) \varepsilon_{k, t} \varepsilon_{\ell, t}\right] h^{2} \\
& -\frac{1}{n f_{U}\left(U_{t}\right)} \sum_{q=1}^{n} K_{h, q}\left(U_{t}\right)\left[\frac{\varepsilon_{\ell, t} \sigma_{k}\left(U_{q}\right) \varepsilon_{k, q}}{\sigma_{k}\left(U_{t}\right)}+\frac{\varepsilon_{k, t} \sigma_{\ell}\left(U_{q}\right) \varepsilon_{\ell, q}}{\sigma_{\ell}\left(U_{t}\right)}\right. \\
& \left.+\left(\frac{\sigma_{k}^{2}\left(U_{q}\right) \xi_{k, q}}{2 \sigma_{k}^{2}\left(U_{t}\right)}+\frac{\sigma_{\ell}^{2}\left(U_{q}\right) \xi_{\ell, q}}{2 \sigma_{\ell}^{2}\left(U_{t}\right)}\right) \varepsilon_{k, t} \varepsilon_{\ell, t}\right]+\delta_{n},
\end{aligned}
$$


by taking conditional expectation at $U_{t}=u$,

$$
\begin{aligned}
= & -\frac{1}{2} w_{2}^{K}\left[\frac{\varrho_{k, \ell}(u) \sigma_{k}^{2^{\prime \prime}}(u)}{2 \sigma_{k}^{2}(u)}+\frac{\varrho_{k, \ell}(u) \sigma_{\ell}^{2^{\prime \prime}}(u)}{2 \sigma_{\ell}^{2}(u)}\right] h^{2}-E\left\{\frac { 1 } { n f _ { U } ( U _ { t } ) } \sum _ { q = 1 } ^ { n } K _ { h , q } ( U _ { t } ) \left[\frac{\varepsilon_{\ell, t} \sigma_{k}\left(U_{q}\right) \varepsilon_{k, q}}{\sigma_{k}\left(U_{t}\right)}\right.\right. \\
& \left.\left.+\frac{\varepsilon_{k, t} \sigma_{\ell}\left(U_{q}\right) \varepsilon_{\ell, q}}{\sigma_{\ell}\left(U_{t}\right)}+\left(\frac{\sigma_{k}^{2}\left(U_{q}\right) \xi_{k, q}}{2 \sigma_{k}^{2}\left(U_{t}\right)}+\frac{\sigma_{\ell}^{2}\left(U_{q}\right) \xi_{\ell, q}}{2 \sigma_{\ell}^{2}\left(U_{t}\right)}\right) \varepsilon_{k, t} \varepsilon_{\ell, t}\right] \mid U_{t}=u\right\}+\delta_{n},
\end{aligned}
$$

for the second part of (A.4) on the right hand side, we focus on the approximation of the first term $E\left\{\frac{1}{n f_{U}\left(U_{t}\right)} \sum_{q=1}^{n} K_{h, q}\left(U_{t}\right) \frac{\varepsilon_{\ell, t} \sigma_{k}\left(U_{q}\right) \varepsilon_{k, q}}{\sigma_{k}\left(U_{t}\right)}\right\}$, and the others could be approximated similarly. For example, for $q=t$,

$$
E\left[\frac{1}{n f_{U}\left(U_{t}\right)} K_{h, q}\left(U_{t}\right) \frac{\varepsilon_{\ell, t} \sigma_{k}\left(U_{q}\right) \varepsilon_{k, q}}{\sigma_{k}\left(U_{t}\right)} \mid U_{t}=u\right]=O\left(\frac{1}{n h}\right),
$$

for $q \neq t$,

$$
E\left[\frac{1}{n f_{U}\left(U_{t}\right)} K_{h, q}\left(U_{t}\right) \frac{\varepsilon_{\ell, t} \sigma_{k}\left(U_{q}\right) \varepsilon_{k, q}}{\sigma_{k}\left(U_{t}\right)} \mid U_{t}=u\right]=0
$$

Therefore,

$$
E\left(\hat{\varepsilon}_{k, t} \hat{\varepsilon}_{\ell, t}-\varepsilon_{k, t} \varepsilon_{\ell, t} \mid U_{t}=u\right)=-\frac{1}{2} w_{2}^{K}\left[\frac{\varrho_{k, \ell}(u) \sigma_{k}^{2^{\prime \prime}}(u)}{2 \sigma_{k}^{2}(u)}+\frac{\varrho_{k, \ell}(u) \sigma_{\ell}^{2^{\prime \prime}}(u)}{2 \sigma_{\ell}^{2}(u)}\right] h^{2}+\delta_{n},
$$

then the following result could be derived by applying (A.3), i.e.

$$
\begin{aligned}
\hat{\varrho}_{k, \ell}(u)-\varrho_{k, \ell}^{*}(u)= & -\frac{1}{2} w_{2}^{K}\left[\frac{\varrho_{k, \ell}(u) \sigma_{k}^{2^{\prime \prime}}(u)}{2 \sigma_{k}^{2}(u)}+\frac{\varrho_{k, \ell}(u) \sigma_{\ell}^{2^{\prime \prime}}(u)}{2 \sigma_{\ell}^{2}(u)}\right] h^{2}-\frac{\varrho_{k, \ell}(u)}{n f_{U}(u)} \\
& \sum_{t=1}^{n} K * K_{h, t}(u)\left[\frac{\sigma_{k}^{2}\left(U_{t}\right) \xi_{k, t}}{2 \sigma_{k}^{2}(u)}+\frac{\sigma_{\ell}^{2}\left(U_{t}\right) \xi_{\ell, t}}{2 \sigma_{\ell}^{2}(u)}\right]+\delta_{n},
\end{aligned}
$$

where $K * K(v)=\int K(x) K(x+v) d x$, and $K * K_{h, t}(u)=\frac{1}{h} K * K\left(\frac{u-U_{t}}{h}\right)$.

Finally,

$$
\begin{aligned}
\hat{\varrho}_{k, \ell}(u)-\varrho_{k, \ell}(u) & =\hat{\varrho}_{k, \ell}(u)-\varrho_{k, \ell}^{*}(u)+\varrho_{k, \ell}^{*}(u)-\varrho_{k, \ell}(u) \\
& =\frac{1}{2} w_{2}^{K} B_{\varrho_{k, \ell}}(u) h^{2}+N_{\varrho}(u)+\delta_{n},
\end{aligned}
$$

where

$$
B_{\varrho_{k, \ell}}(u)=\varrho_{k, \ell}^{\prime \prime}(u)-\varrho_{k, \ell}(u)\left(\frac{\sigma_{k}^{2^{\prime \prime}}(u)}{2 \sigma_{k}^{2}(u)}+\frac{\sigma_{\ell}^{2^{\prime \prime}}(u)}{2 \sigma_{\ell}^{2}(u)}\right),
$$


6

$$
N_{\varrho}(u)=\frac{1}{n f_{U}(u)} \sum_{t=1}^{n}\left[K_{h, t}(u) \epsilon_{k, \ell, t}-K * K_{h, t}(u) \varrho_{k, \ell}(u)\left(\frac{\sigma_{k}^{2}\left(U_{t}\right) \xi_{k, t}}{2 \sigma_{k}^{2}(u)}+\frac{\sigma_{\ell}^{2}\left(U_{t}\right) \xi_{\ell, t}}{2 \sigma_{\ell}^{2}(u)}\right)\right] .
$$

LEMma A.2. Suppose that all assumptions are fulfilled, then for particular $k$ and $\ell$, as $n \rightarrow \infty$, we have uniformly,

$$
\begin{aligned}
& \varrho_{k, \ell}(u)-\varrho_{k, \ell}(u) \\
= & \frac{1}{2} w_{2}^{K}\left[\varrho_{k, \ell}^{\prime \prime}(u)-\varrho_{k, \ell}(u)\left(\frac{\sigma_{k}^{2^{\prime \prime}}(u)}{2 \sigma_{k}^{2}(u)}+\frac{\sigma_{\ell}^{2^{\prime \prime}}(u)}{2 \sigma_{\ell}^{2}(u)}\right)\right] h^{2}+\frac{1}{n f_{U}(u)} \sum_{t=1}^{n} K_{h, t}(u) \epsilon_{k, \ell, t} \\
& -\frac{\varrho_{k, \ell}(u)}{n f_{U}(u)} \sum_{t=1}^{n} K * K_{h, t}(u)\left[\frac{\sigma_{k}^{2}\left(U_{t}\right) \xi_{k, t}}{2 \sigma_{k}^{2}(u)}+\frac{\sigma_{\ell}^{2}\left(U_{t}\right) \xi_{\ell, t}}{2 \sigma_{\ell}^{2}(u)}\right]+O_{p}\left(\delta_{n}\right),
\end{aligned}
$$

where $K_{h, t}=K_{h}\left(U_{t}-u\right), K * K_{h, t}(u)=\frac{1}{h} K * K\left(\frac{u-U_{t}}{h}\right)$, and $\delta_{n}=o_{P}\left(h^{2}+\{\log n /(n h)\}^{1 / 2}\right)$.

Proof of Lemma A.2. The proof of this lemma could be found from the derivation of (A.5).

Proof of Theorem 3.1. By Lemma A.2,

$$
\hat{\varrho}_{k, \ell}(u)=\varrho_{k, \ell}(u)+\frac{1}{2} w_{2}^{K} B_{\hat{\varrho}_{k, \ell}}(u) h^{2}+N_{\hat{\varrho}}(u)+\delta_{n},
$$

where

$$
\begin{aligned}
B_{\varrho_{k, \ell}}(u) & =\varrho_{k, \ell}^{\prime \prime}(u)-\varrho_{k, \ell}(u)\left(\frac{\sigma_{k}^{2^{\prime \prime}}(u)}{2 \sigma_{k}^{2}(u)}+\frac{\sigma_{\ell}^{2^{\prime \prime}}(u)}{2 \sigma_{\ell}^{2}(u)}\right) \\
N_{\hat{\varrho}}(u) & =\frac{1}{n f_{U}(u)} \sum_{t=1}^{n}\left[K_{h, t}(u) \epsilon_{k, \ell, t}-K * K_{h, t}(u) \varrho_{k, \ell}(u)\left(\frac{\sigma_{k}^{2}\left(U_{t}\right) \xi_{k, t}}{2 \sigma_{k}^{2}(u)}+\frac{\sigma_{\ell}^{2}\left(U_{t}\right) \xi_{\ell, t}}{2 \sigma_{\ell}^{2}(u)}\right)\right] .
\end{aligned}
$$

For simplicity, let

$$
Z_{n, t}(u)=K_{h, t}(u) \epsilon_{k, \ell, t}-K * K_{h, t}(u) \varrho_{k, \ell}(u)\left(\frac{\sigma_{k}^{2}\left(U_{t}\right) \xi_{k, t}}{2 \sigma_{k}^{2}(u)}+\frac{\sigma_{\ell}^{2}\left(U_{t}\right) \xi_{\ell, t}}{2 \sigma_{\ell}^{2}(u)}\right),
$$

then

$$
\hat{\varrho}_{k, \ell}(u)-\varrho_{k, \ell}(u)-\frac{1}{2} w_{2}^{K} B_{\hat{\varrho}_{k, \ell}}(u) h^{2}=\frac{1}{n f_{U}(u)} \sum_{t=1}^{n} Z_{n, t}(u)+\delta_{n} .
$$

Based on the above formula,

$$
E\left\{N_{\hat{\varrho}}(u)\right\}=E\left\{\frac{1}{n f_{U}(u)} \sum_{t=1}^{n} Z_{n, t}(u)\right\}
$$




$$
\operatorname{Var}\left\{N_{\hat{\varrho}}(u)\right\}=\frac{1}{n f_{U}^{2}(u)} \operatorname{Var}\left\{Z_{n, 1}(u)\right\}+\frac{2}{n f_{U}^{2}(u)} \sum_{s=1}^{n-1}\left(1-\frac{s}{n}\right) \operatorname{Cov}\left(Z_{n, 1}(u), Z_{n, s+1}(u)\right) .
$$

According to the assumptions, $E\left\{\epsilon_{k, \ell, t} \mid U_{t}\right\}=0, E\left\{\xi_{k, t} \mid U_{t}\right\}=0, E\left\{\xi_{\ell, t} \mid U_{t}\right\}=0$, then $E\left\{Z_{n, t}(u) \mid U_{t}\right\}=0, E\left\{N_{\hat{\varrho}}(u)\right\}=0$, and

$$
\begin{aligned}
E\left\{Z_{n, t}^{2}(u) \mid U_{t}\right\}= & K_{h, t}^{2}(u) E\left\{\epsilon_{k, \ell, t}^{2} \mid U_{t}\right\}+K * K_{h, t}^{2}(u) \varrho_{k, \ell}^{2}(u)\left[\frac{\sigma_{k}^{4}\left(U_{t}\right) E\left\{\xi_{k, t}^{2} \mid U_{t}\right\}}{4 \sigma_{k}^{4}(u)}+\frac{\sigma_{\ell}^{4}\left(U_{t}\right) E\left\{\xi_{\ell, t}^{2} \mid U_{t}\right\}}{4 \sigma_{\ell}^{4}(u)}\right. \\
& \left.+\frac{\sigma_{k}^{2}\left(U_{t}\right) \sigma_{\ell}^{2}\left(U_{t}\right) E\left\{\xi_{k, t} \xi_{\ell, t} \mid U_{t}\right\}}{2 \sigma_{k}^{2}(u) \sigma_{\ell}^{2}(u)}\right]-K_{h, t}(u) K * K_{h, t}(u) \varrho_{k, \ell}(u) \\
& {\left[\frac{\sigma_{k}^{2}\left(U_{t}\right) E\left\{\xi_{k, t} \epsilon_{k, \ell, t} \mid U_{t}\right\}}{\sigma_{k}^{2}(u)}+\frac{\sigma_{\ell}^{2}\left(U_{t}\right) E\left\{\xi_{\ell, t} \epsilon_{k, \ell, t} \mid U_{t}\right\}}{\sigma_{\ell}^{2}(u)}\right] }
\end{aligned}
$$

then

$$
E\left\{Z_{n, t}^{2}(u)\right\}=\frac{f_{U}(u)}{h}\left[\nu_{K}^{2} \zeta_{\epsilon}^{k, \ell}(u)+\frac{1}{4} \nu_{K * K}^{2} \varrho_{k, \ell}^{2}(u) \zeta_{\xi}^{k, \ell}(u)-\varrho_{k, \ell}(u) \nu_{K, K * K} \zeta_{\epsilon, \xi}^{k, \ell}(u)\right]+o\left(\frac{1}{h}\right),
$$

where

$$
\begin{aligned}
& \zeta_{\epsilon}^{k, \ell}(u)=E\left\{\epsilon_{k, \ell, t}^{2} \mid U_{t}=u\right\}, \zeta_{\xi}^{k, \ell}(u)=E\left\{\left(\xi_{k, t}+\xi_{\ell, t}\right)^{2} \mid U_{t}=u\right\} \\
& \zeta_{\epsilon, \zeta}^{k, \ell}(u)=E\left\{\epsilon_{k, \ell, t}\left(\xi_{k, t}+\xi_{\ell, t}\right) \mid U_{t}=u\right\} .
\end{aligned}
$$

Let $d_{n} \rightarrow \infty$ be a sequence of integers such that $h d_{n} \rightarrow 0$. Define

$$
\mathcal{Z}_{1}=\sum_{s=1}^{d_{n}-1}\left|\operatorname{Cov}\left(Z_{n, 1}(u), Z_{n, s+1}(u)\right)\right|, \quad \mathcal{Z}_{2}=\sum_{s=d_{n}}^{n-1}\left|\operatorname{Cov}\left(Z_{n, 1}(u), Z_{n, s+1}(u)\right)\right| .
$$

Conditioning on $\left(U_{1}, U_{s+1}\right)$, and by (C2), (C4) and (C5),

$$
\begin{aligned}
& \left|\operatorname{Cov}\left(Z_{n, 1}(u), Z_{n, s+1}(u)\right)\right| \\
= & E\left\{\frac{1}{h^{2}}\left[K\left(\frac{U_{1}-u}{h}\right) \epsilon_{k, \ell, 1}-K * K\left(\frac{U_{1}-u}{h}\right) \varrho_{k, \ell}(u)\left(\frac{\sigma_{k}^{2}\left(U_{1}\right) \xi_{k, 1}}{2 \sigma_{k}^{2}(u)}+\frac{\sigma_{\ell}^{2}\left(U_{1}\right) \xi_{\ell, 1}}{2 \sigma_{\ell}^{2}(u)}\right)\right]\right. \\
& {\left.\left[K\left(\frac{U_{s+1}-u}{h}\right) \epsilon_{k, \ell, s+1}-K * K\left(\frac{U_{s+1}-u}{h}\right) \varrho_{k, \ell}(u)\left(\frac{\sigma_{k}^{2}\left(U_{s+1}\right) \xi_{k, s+1}}{2 \sigma_{k}^{2}(u)}+\frac{\sigma_{\ell}^{2}\left(U_{s+1}\right) \xi_{\ell, s+1}}{2 \sigma_{\ell}^{2}(u)}\right)\right]\right\} } \\
\leq & C L_{2}
\end{aligned}
$$

for some generic constant $C>0$. Then it follows that $\mathcal{Z}_{1} \leq d_{n} C L_{2}$. We now consider the contribution of $\mathcal{Z}_{2}$. For this $\alpha$-mixing process, by Davydov's lemma,

$$
\left|\operatorname{Cov}\left(Z_{n, 1}(u), Z_{n, s+1}(u)\right)\right|=E\left|\left(Z_{n, 1}(u) Z_{n, s+1}(u)\right)\right| \leq 8[\alpha(s)]^{\frac{\delta}{1+\delta}}\left\{E\left|Z_{n, 1}(u)\right|^{2(1+\delta)}\right\}^{\frac{1}{1+\delta}} .
$$


By conditioning on $U_{1}$, and using (C2) and (C3),

$$
\begin{aligned}
E\left|Z_{n, 1}(u)\right|^{2(1+\delta)} & =E\left|K_{h, 1}(u) \epsilon_{k, \ell, 1}-K * K_{h, 1}(u) \varrho_{k, \ell}(u)\left(\frac{\sigma_{k}^{2}\left(U_{1}\right) \xi_{k, 1}}{2 \sigma_{k}^{2}(u)}+\frac{\sigma_{\ell}^{2}\left(U_{1}\right) \xi_{\ell, 1}}{2 \sigma_{\ell}^{2}(u)}\right)\right|^{2(1+\delta)} \\
& \leq C L_{2} h^{-2(1+\delta)+1} .
\end{aligned}
$$

Hence, for $\frac{\delta}{1+\delta}<\gamma<1$,

$$
\begin{aligned}
\mathcal{Z}_{2} & \leq \sum_{s=d_{n}}^{n-1} 8[\alpha(s)]^{\frac{\delta}{1+\delta}}\left\{E\left|\tilde{Z}_{n, 1}(u)\right|^{2(1+\delta)}\right\}^{\frac{1}{1+\delta}} \leq\left(C L_{2}\right)^{\frac{1}{1+\delta}} 8\left(h^{-2(1+\delta)+1}\right)^{\frac{1}{1+\delta}} \sum_{s=d_{n}}^{\infty}\left[s^{-\beta}\right]^{\frac{\delta}{1+\delta}} \\
& \leq M h^{-2+\frac{1}{1+\delta}} \sum_{s=d_{n}}^{\infty} s^{-2}=M h^{-2+\frac{1}{1+\delta}} d_{n}^{-\gamma} \sum_{s=d_{n}}^{\infty} s^{-2+\gamma}=o(1 / h)
\end{aligned}
$$

by taking $h^{-1+\frac{1}{1+\delta}} d_{n}^{-\gamma}=1$. Together with the above results,

$$
\sum_{s=1}^{n-1} \operatorname{Cov}\left(Z_{n, 1}(u), Z_{n, s+1}(u)\right)=o(1 / h),
$$

Thus,

$\operatorname{Var}\left\{N_{\hat{\varrho}}(u)\right\}=\frac{1}{n h f_{U}(u)}\left[\nu_{K}^{2} \zeta_{\epsilon}^{k, \ell}(u)+\frac{1}{4} \nu_{K * K}^{2} \varrho_{k, \ell}^{2}(u) \zeta_{\xi}^{k, \ell}(u)-\varrho_{k, \ell}(u) \nu_{K, K * K} \zeta_{\epsilon, \xi}^{k, \ell}(u)\right]+o\left(\frac{1}{n h}\right)$.

Therefore, the following asymptotic normality could be obtained accordingly,

$$
(n h)^{1 / 2}\left\{\hat{\varrho}_{k, \ell}(u)-\varrho_{k, \ell}(u)-\frac{1}{2} w_{2}^{K} B_{\hat{\varrho}_{k, \ell}}(u) h^{2}\right\} \rightarrow N\left(0, f_{U}^{-1}(u) \omega_{2, k, \ell}(u)\right),
$$

where

$$
\begin{gathered}
B_{\varrho_{k, \ell}}(u)=\varrho_{k, \ell}^{\prime \prime}(u)-\varrho_{k, \ell}(u)\left(\frac{\sigma_{k}^{2^{\prime \prime}}(u)}{2 \sigma_{k}^{2}(u)}+\frac{\sigma_{\ell}^{2^{\prime \prime}}(u)}{2 \sigma_{\ell}^{2}(u)}\right), \\
\omega_{2, k, \ell}(u)=\nu_{K}^{2} \zeta_{\epsilon}^{k, \ell}(u)+\frac{1}{4} \nu_{K * K}^{2} \varrho_{k, \ell}^{2}(u) \zeta_{\xi}^{k, \ell}(u)-\varrho_{k, \ell}(u) \nu_{K, K * K} \zeta_{\epsilon, \xi}^{k, \ell}(u) .
\end{gathered}
$$

Proof of Theorem 3.2. From section 2.4, local linear method is applied to estimate $G_{k \ell}(u)$,

$$
\hat{G}_{k \ell}(u)=\frac{\sum_{t=1}^{n}\left(\hat{\varepsilon}_{k, t} \hat{\varepsilon}_{\ell, t}-\hat{a}_{k \ell}\right) W_{n, h}\left(U_{t}-u\right)}{\sum_{t=1}^{n} W_{n, h}\left(U_{t}-u\right)}=\hat{\varrho}_{k, \ell}(u)-\hat{a}_{k \ell},
$$


By (A.5), together with the definition of $\hat{a}_{k \ell}$ as well as (??), for a particular $G_{k \ell}(u)$, under the regularity conditions, we could have uniformly for $u \in[c, d]$,

$$
\begin{aligned}
\hat{G}_{k \ell}(u)= & G_{k \ell}(u)+\hat{\varrho}_{k, \ell}(u)-\varrho_{k, \ell}(u)-\hat{a}_{k \ell}+a_{k \ell} \\
= & G_{k \ell}(u)+\frac{1}{2} w_{2}^{K} h^{2} B_{\hat{\varrho}_{k, \ell}}(u)+N_{\hat{\varrho}}(u)-\frac{1}{2} w_{2}^{K} h^{2}\left(\frac{1}{n} \sum_{t=1}^{n} B_{\hat{\varrho}_{k, \ell}}\left(U_{t}\right)\right) \\
& -\frac{1}{n} \sum_{t=1}^{n} N_{\hat{\varrho}}\left(U_{t}\right)-\frac{1}{n} \sum_{t=1}^{n} G_{k \ell}\left(U_{t}\right)+\delta_{n},
\end{aligned}
$$

where $\delta_{n}=o_{P}\left(h^{2}+\{\log n /(n h)\}^{1 / 2}\right)$.

Let $K_{f}(u)=\left(\frac{K_{h, 1}(u)}{f_{U}(u)}, \ldots, \frac{K_{h, n}(u)}{f_{U}(u)}\right)^{\top}, K * K_{f}(u)=\left(\frac{K * K_{h, 1}(u)}{f_{U}(u)}, \ldots, \frac{K * K_{h, n}(u)}{f_{U}(u)}\right)^{\top}$,

$$
\varrho(u)=\left(\begin{array}{c}
\varrho_{1,2}(u) \\
\vdots \\
\varrho_{1, m}(u) \\
\varrho_{2,3}(u) \\
\vdots \\
\varrho_{2, m}(u) \\
\vdots \\
\left.\varrho_{m-1, m}(u)\right)
\end{array}\right), \varrho^{\prime \prime}(u)=\left(\begin{array}{c}
\varrho_{1,2}^{\prime \prime}(u) \\
\vdots \\
\varrho_{1, m}^{\prime \prime}(u) \\
\varrho_{2,3}^{\prime \prime}(u) \\
\vdots \\
\varrho_{2, m}^{\prime \prime}(u) \\
\vdots \\
\left.\varrho_{m-1, m}^{\prime \prime}(u)\right)
\end{array}\right), \sigma(u)=\left(\begin{array}{c}
\frac{\sigma_{1}^{2 \prime \prime}(u)}{2 \sigma_{1}^{2}(u)}+\frac{\sigma_{2}^{2^{\prime \prime}}(u)}{2 \sigma_{2}^{2}(u)} \\
\vdots \\
\frac{\sigma_{1}^{2 \prime \prime}(u)}{2 \sigma_{1}^{2}(u)}+\frac{\sigma_{m}^{2 \prime \prime}(u)}{2 \sigma_{m}^{2}(u)} \\
\frac{\sigma_{2}^{2 \prime \prime}(u)}{2 \sigma_{2}^{2}(u)}+\frac{\sigma_{3}^{2 \prime}(u)}{2 \sigma_{3}^{2}(u)} \\
\vdots \\
\frac{\sigma_{2}^{2 \prime \prime}(u)}{2 \sigma_{2}^{2}(u)}+\frac{\sigma_{m}^{2 \prime \prime}(u)}{2 \sigma_{m}^{2}(u)} \\
\vdots \\
\frac{\sigma_{m-1}^{2 \prime \prime}(u)}{2 \sigma_{m-1}^{2}(u)}+\frac{\sigma_{m}^{2 \prime}(u)}{2 \sigma_{m}^{2}(u)}
\end{array}\right),
$$

and

$$
\boldsymbol{\epsilon}=\left(\begin{array}{ccc}
\epsilon_{1,2,1} & \cdots & \epsilon_{1,2, n} \\
\vdots & & \vdots \\
\epsilon_{1, m, 1} & \cdots & \epsilon_{1, m, n} \\
\epsilon_{2,3,1} & \cdots & \epsilon_{2,3, n} \\
\vdots & & \vdots \\
\epsilon_{2, m, 1} & \cdots & \epsilon_{2, m, n} \\
\vdots & & \vdots \\
\epsilon_{m-1, m, 1} & \cdots & \epsilon_{m-1, m, n}
\end{array}\right)=\left(\tilde{\epsilon}_{1}, \ldots, \tilde{\epsilon}_{n}\right), \boldsymbol{\xi}=\left(\begin{array}{ccc}
\xi_{1,1}+\xi_{2,1} & \cdots & \xi_{1, n}+\xi_{2, n} \\
\vdots & & \vdots \\
\xi_{1,1}+\xi_{m, 1} & \cdots & \xi_{1, n}+\xi_{m, n} \\
\xi_{2,1}+\xi_{3,1} & \cdots & \xi_{2, n}+\xi_{3, n} \\
\vdots & & \vdots \\
\xi_{2,1}+\xi_{m, 1} & \cdots & \xi_{2, n}+\xi_{m, n} \\
\vdots & & \vdots \\
\xi_{m-1,1}+\xi_{m, 1} & \cdots & \xi_{m-1, n}+\xi_{m, n}
\end{array}\right)=\left(\tilde{\xi}_{1}, . ., \tilde{\xi}_{n}\right)
$$




$$
\boldsymbol{\sigma}_{\xi}(u)=\left(\begin{array}{ccc}
\frac{\sigma_{1}^{2}\left(U_{1}\right) \xi_{1,1}}{\sigma_{1}^{2}(u)}+\frac{\sigma_{2}^{2}\left(U_{1}\right) \xi_{2,1}}{\sigma_{2}^{2}(u)} & \cdots & \frac{\sigma_{1}^{2}\left(U_{n}\right) \xi_{1, n}}{\sigma_{1}^{2}(u)}+\frac{\sigma_{2}^{2}\left(U_{n}\right) \xi_{2, n}}{\sigma_{2}^{2}(u)} \\
\vdots & & \vdots \\
\frac{\sigma_{1}^{2}\left(U_{1}\right) \xi_{1,1}}{\sigma_{1}^{2}(u)}+\frac{\sigma_{m}^{2}\left(U_{1}\right) \xi_{m, 1}}{\sigma_{m}^{2}(u)} & \cdots & \frac{\sigma_{1}^{2}\left(U_{n}\right) \xi_{1, n}}{\sigma_{1}^{2}(u)}+\frac{\sigma_{m}^{2}\left(U_{n}\right) \xi_{m, n}}{\sigma_{m}^{2}(u)} \\
\frac{\sigma_{2}^{2}\left(U_{1}\right) \xi_{2,1}}{\sigma_{2}^{2}(u)}+\frac{\sigma_{3}^{2}\left(U_{1}\right) \xi_{3,1}}{\sigma_{3}^{2}(u)} & \cdots & \frac{\sigma_{2}^{2}\left(U_{n}\right) \xi_{2, n}}{\sigma_{2}^{2}(u)}+\frac{\sigma_{3}^{2}\left(U_{n}\right) \xi_{3, n}}{\sigma_{3}^{2}(u)} \\
\vdots & & \vdots \\
\frac{\sigma_{2}^{2}\left(U_{1}\right) \xi_{2,1}}{\sigma_{2}^{2}(u)}+\frac{\sigma_{m}^{2}\left(U_{1}\right) \xi_{m, 1}}{\sigma_{m}^{2}(u)} & \cdots & \frac{\sigma_{2}^{2}\left(U_{n}\right) \xi_{2, n}}{\sigma_{2}^{2}(u)}+\frac{\sigma_{m}^{2}\left(U_{n}\right) \xi_{m, n}}{\sigma_{m}^{2}(u)} \\
\vdots & \vdots \\
\frac{\sigma_{m-1}^{2}\left(U_{1}\right) \xi_{2,1}}{\sigma_{m-1}^{2}(u)}+\frac{\sigma_{m}^{2}\left(U_{1}\right) \xi_{m, 1}}{\sigma_{m}^{2}(u)} & \cdots & \frac{\sigma_{m-1}^{2}\left(U_{n}\right) \xi_{m-1, n}}{\sigma_{m-1}^{2}(u)}+\frac{\sigma_{m}^{2}\left(U_{n}\right) \xi_{m, n}}{\sigma_{m}^{2}(u)}
\end{array}\right)=\left(\tilde{\sigma}_{\xi, 1}(u), \ldots, \tilde{\sigma}_{\xi, n}(u)\right),
$$

therefore,

$$
\begin{aligned}
\hat{\mathrm{G}}(u)= & \mathrm{G}(u)+\frac{1}{2} w_{2}^{K} h^{2}\left[\varrho^{\prime \prime}(u)-\frac{1}{2} \varrho(u) \circ \sigma(u)\right]+\frac{1}{n} \boldsymbol{\epsilon} K_{f}(u)-\frac{1}{2 n} \varrho(u) \circ\left(\boldsymbol{\sigma}_{\xi}(u) K * K_{f}(u)\right) \\
& -\frac{1}{2} w_{2}^{K} h^{2}\left[\frac{1}{n} \sum_{t=1}^{n} \varrho^{\prime \prime}\left(U_{t}\right)-\frac{1}{2 n} \sum_{t=1}^{n} \varrho\left(U_{t}\right) \circ \sigma\left(U_{t}\right)\right]-\frac{1}{n} \sum_{t=1}^{n} \mathrm{G}\left(U_{t}\right) \\
(\text { A.6 } \quad & -\frac{1}{n} \sum_{t=1}^{n}\left[\frac{1}{n} \boldsymbol{\epsilon} K_{f}\left(U_{t}\right)-\frac{1}{2 n} \varrho\left(U_{t}\right) \circ\left(\boldsymbol{\sigma}_{\xi}\left(U_{t}\right) K * K_{f}\left(U_{t}\right)\right)\right]+\delta_{n},
\end{aligned}
$$

and

$$
\begin{aligned}
\hat{\mathbf{G}}= & \mathbf{G}+\frac{1}{2} w_{2}^{K} h^{2}\left(\varrho^{\prime \prime}-\frac{1}{2} \boldsymbol{\varrho} \circ \boldsymbol{\sigma}\right)+\frac{1}{n} \boldsymbol{\epsilon} \mathbf{K}_{f}-\frac{1}{2 n} \varrho \circ\left(\boldsymbol{\sigma}_{\xi} \mathbf{K} * \mathbf{K}_{f}\right)-\frac{1}{n} \mathbf{G} 1_{n} 1_{n}^{\top} \\
& -\frac{1}{2} w_{2}^{K} h^{2}\left(\frac{1}{n} \varrho^{\prime \prime} 1_{n} 1_{n}^{\top}-\frac{1}{2 n}(\varrho \circ \boldsymbol{\sigma}) 1_{n} 1_{n}^{\top}\right)-\frac{1}{n}\left[\frac{1}{n} \boldsymbol{\epsilon} \mathbf{K}_{f} 1_{n} 1_{n}^{\top}-\frac{1}{2 n}\left(\varrho \circ\left(\boldsymbol{\sigma}_{\xi} \mathbf{K} * \mathbf{K}_{f}\right)\right) 1_{n} 1_{n}^{\top}\right] \\
& +\delta_{n} \\
= & \mathbf{G}+\tilde{\mathcal{E}}_{n},
\end{aligned}
$$

where $\circ$ denotes the hadamard product of two matrices, $\varrho, \varrho^{\prime \prime}, \boldsymbol{\sigma}$ are $M \times n$ matrices, i.e. $\varrho=\left(\varrho\left(U_{1}\right), \ldots, \varrho\left(U_{n}\right)\right), \varrho^{\prime \prime}=\left(\varrho^{\prime \prime}\left(U_{1}\right), \ldots, \varrho^{\prime \prime}\left(U_{n}\right)\right), \boldsymbol{\sigma}=\left(\sigma\left(U_{1}\right), \ldots, \sigma\left(U_{n}\right)\right), \mathbf{K}_{f}$ is a $n \times n$ matrix, $\mathbf{K} * \mathbf{K}_{f}$ is a $n^{2} \times n$ matrix, and $\boldsymbol{\sigma}_{\xi}$ is a $M \times n^{2}$ matrix, i.e. $\mathbf{K}_{f}=\left(K_{f}\left(U_{1}\right), \ldots, K_{f}\left(U_{n}\right)\right)$, $\mathbf{K} * \mathbf{K}_{f}=\operatorname{diag}\left(K * K_{f}\left(U_{1}\right), \ldots, K * K_{f}\left(U_{n}\right)\right)$, and $\boldsymbol{\sigma}_{\xi}=\left(\boldsymbol{\sigma}_{\xi}\left(U_{1}\right), \ldots, \boldsymbol{\sigma}_{\xi}\left(U_{n}\right)\right)$.

Recall $\varrho_{k, \ell}(u)=a_{k \ell}+G_{k \ell}(u)$ by $(2.7)$, then $\varrho=a 1_{M} 1_{n}^{\top}+\mathbf{G}, \varrho^{\prime \prime}=\mathbf{G}^{\prime \prime}$, therefore,

$$
\begin{aligned}
\mathbf{E}_{n} & =\boldsymbol{\Lambda}_{\hat{\mathbf{G}}}-\boldsymbol{\Lambda} \\
& =\frac{1}{n} \hat{\mathbf{G}} \hat{\mathbf{G}}^{\top}-E\left\{G(U) G(U)^{\top}\right\}=\frac{1}{n}\left(\mathbf{G}+\tilde{\mathcal{E}}_{n}\right)\left(\mathbf{G}+\tilde{\mathcal{E}}_{n}\right)^{\top}-E\left\{G(U) G(U)^{\top}\right\} \\
& =\left(\frac{1}{2} w_{2}^{K} h^{2}\right) \frac{1}{n}\left[\mathbf{G}^{\prime \prime} \mathbf{G}^{\top}+\mathbf{G G}^{\prime \prime}{ }^{\top}-\frac{1}{2}(\varrho \circ \boldsymbol{\sigma}) \mathbf{G}^{\top}-\frac{1}{2} \mathbf{G}\left(\varrho^{\top} \circ \boldsymbol{\sigma}^{\top}\right)\right]+\frac{1}{n^{2}}\left(\boldsymbol{\epsilon} \mathbf{K}_{f} \mathbf{G}^{\top}+\mathbf{G K}_{f}^{\top} \boldsymbol{\epsilon}^{\top}\right)
\end{aligned}
$$




$$
\begin{aligned}
& -\frac{1}{2 n^{2}}\left[\left(\varrho \circ\left(\boldsymbol{\sigma}_{\xi} \mathbf{K} * \mathbf{K}_{f}\right)\right) \mathbf{G}^{\top}+\mathbf{G}\left(\varrho^{\top} \circ\left(\mathbf{K} * \mathbf{K}_{f}^{\top} \boldsymbol{\sigma}_{\xi}^{\top}\right)\right)\right]+\frac{1}{n} \mathbf{G G}^{\top}-E\left\{G(U) G(U)^{\top}\right\} \\
& +o_{p}\left(\frac{1}{\sqrt{n}}\right),
\end{aligned}
$$

due to the fact that $h=O\left(n^{-\frac{1}{5}}\right)$ as $n \rightarrow \infty$, and $E G_{k \ell}(U)=0, \sum_{t=1}^{n} G_{k \ell}\left(U_{t}\right)=O_{p}(\sqrt{n})$,

$$
\mathbf{G} 1_{n}=O_{p}(\sqrt{n}), \quad \mathbf{G}^{\prime \prime} 1_{n}=O_{p}(n)
$$

Note that under condition (C5), $K(\cdot)$ is a bounded function with a bounded support, satisfying the Lipschitz condition, then $K * K(\cdot)$ is also bounded with bounded support, and Lipschitz continuous. Note that by (C1), (C2) and (C4), $E\left|G_{k \ell}(U) / f(U)\right|^{2+\delta}<\infty$, $E\left|\frac{\varrho_{k_{1}, \ell_{1}}(U) G_{k_{2} \ell_{2}}(U)}{2 \sigma_{k_{1}}^{2}(U) f_{U}(U)}\right|^{2+\delta}<\infty, E\left|\frac{\varrho_{k_{1}, \ell_{1}}(U) G_{k_{2} \ell_{2}}(U)}{2 \sigma_{\ell_{1}}^{2}(U) f_{U}(U)}\right|^{2+\delta}<\infty$, for particular $k, \ell$ and $k_{1}, \ell_{1}, k_{2}$, $\ell_{2}$, thus the following equations hold uniformly for $u \in[c, d]$,

$$
\begin{aligned}
& \left|\frac{1}{n} \sum_{q=1}^{n} G_{k \ell}\left(U_{q}\right) \frac{K_{h}\left(u-U_{q}\right)}{f\left(U_{q}\right)}-G_{k \ell}(u)\right|=O_{p}\left(h^{2}+\left(\frac{\log n}{n h}\right)^{\frac{1}{2}}\right), \\
& \left|\frac{1}{n} \sum_{q=1}^{n} \frac{\varrho_{k_{1}, \ell_{1}}\left(U_{q}\right) G_{k_{2}, \ell_{2}}\left(U_{q}\right)}{2 \sigma_{k_{1}}^{2}\left(U_{q}\right) f_{U}\left(U_{q}\right)} K * K_{h}\left(u-U_{q}\right)-\frac{\varrho_{k_{1}, \ell_{1}}(u) G_{k_{2}, \ell_{2}}(u)}{2 \sigma_{k_{1}}^{2}(u)}\right|=O_{p}\left(h^{2}+\left(\frac{\log n}{n h}\right)^{\frac{1}{2}}\right), \\
& \left|\frac{1}{n} \sum_{q=1}^{n} \frac{\varrho_{k_{1}, \ell_{1}}\left(U_{q}\right) G_{k_{2}, \ell_{2}}\left(U_{q}\right)}{2 \sigma_{\ell_{1}}^{2}\left(U_{q}\right) f_{U}\left(U_{q}\right)} K * K_{h}\left(u-U_{q}\right)-\frac{\varrho_{k_{1}, \ell_{1}}(u) G_{k_{2}, \ell_{2}}(u)}{2 \sigma_{\ell_{1}}^{2}(u)}\right|=O_{p}\left(h^{2}+\left(\frac{\log n}{n h}\right)^{\frac{1}{2}}\right),
\end{aligned}
$$

then the following term could be approximated accordingly,

$$
\begin{aligned}
& \frac{1}{n^{2}} \mathbf{G K}_{f}^{\top} \boldsymbol{\epsilon}^{\top}=\frac{1}{n} \mathbf{G} \boldsymbol{\epsilon}^{\top}+o_{p}\left(\frac{1}{\sqrt{n}}\right), \\
& \frac{1}{2 n^{2}} \mathbf{G}\left(\boldsymbol{\varrho}^{\top} \circ\left(\mathbf{K} * \mathbf{K}_{f}^{\top} \boldsymbol{\sigma}_{\xi}^{\top}\right)\right)=\frac{1}{2 n} \mathbf{G}\left(\varrho^{\top} \circ \boldsymbol{\xi}^{\top}\right)+o_{p}\left(\frac{1}{\sqrt{n}}\right) .
\end{aligned}
$$

Therefore,

$$
\mathbf{E}_{n}=\boldsymbol{\Lambda}_{\hat{\mathbf{G}}}-\boldsymbol{\Lambda}=\left(\frac{1}{2} w_{2}^{K} h^{2}\right) \mathbf{W}_{n}+\mathbf{H}_{n 1}+\mathbf{H}_{n 1}^{\top}+\mathbf{H}_{n 2}+o_{p}\left(\frac{1}{\sqrt{n}}\right) 1_{\frac{m(m-1)}{2}} 1_{\frac{m(m-1)}{2}}^{\top}
$$

where

$$
\begin{aligned}
\mathbf{W}_{n} & =\frac{1}{n}\left[\mathbf{G G}^{\prime \prime \top}+\mathbf{G}^{\prime \prime} \mathbf{G}^{\top}-\frac{1}{2} \mathbf{G}\left(\varrho^{\top} \circ \boldsymbol{\sigma}^{\top}\right)-\frac{1}{2}(\varrho \circ \boldsymbol{\sigma}) \mathbf{G}^{\top}\right], \\
\mathbf{H}_{n 1} & =\frac{1}{n} \mathbf{G}\left[\boldsymbol{\epsilon}^{\top}-\frac{1}{2}\left(\varrho^{\top} \circ \boldsymbol{\xi}^{\top}\right)\right] \\
\mathbf{H}_{n 2} & =\frac{1}{n} \mathbf{G G}^{\top}-E\left\{\mathrm{G}(U) \mathrm{G}(U)^{\top}\right\} .
\end{aligned}
$$


Because $\boldsymbol{\Lambda}$ is a real symmetric matrix, and $V_{j}$ is the normalized eigenvector associated with a simple eigenvalue $\lambda_{j}$ of $\boldsymbol{\Lambda}$ for $j=1, \ldots, p$. Then by the results in Magnus (1985), a realvalued function $\mathfrak{u}_{j}$ and a vector function $\mathcal{V}_{j}(j=1, \ldots, p)$ are defined for all $\boldsymbol{\Lambda}^{*}$ in some neighbourhood $N(\boldsymbol{\Lambda})$ of $\boldsymbol{\Lambda}$ such that

$$
\begin{gathered}
\mathfrak{u}_{j}(\boldsymbol{\Lambda})=\lambda_{j}, \quad \mathcal{V}_{j}(\boldsymbol{\Lambda})=\mathrm{V}_{j}, \quad \mathfrak{u}_{j}\left(\boldsymbol{\Lambda}_{\hat{\mathrm{G}}}\right)=\hat{\lambda}_{j}, \quad \mathcal{V}_{j}\left(\boldsymbol{\Lambda}_{\hat{\mathbf{G}}}\right)=\hat{\mathrm{V}}_{j} \\
\boldsymbol{\Lambda}^{*} \mathcal{V}_{j}=\mathfrak{u}_{j} \mathcal{V}_{j}, \quad \mathcal{V}_{j}^{\top} \mathcal{V}_{j}=1, \quad \boldsymbol{\Lambda}^{*} \in N(\boldsymbol{\Lambda}) .
\end{gathered}
$$

Moreover, the functions $\mathfrak{u}_{j}$ and $\mathcal{V}_{j}$ are $\infty$ times differentiable, and the differentials at $\boldsymbol{\Lambda}$ are

$$
\begin{aligned}
& d \mathfrak{u}_{j}=\mathrm{V}_{j}^{\top} d \boldsymbol{\Lambda}^{*} \mathrm{~V}_{j}, \\
& d \mathcal{V}_{j}=\left(\lambda_{j} \mathbf{I}-\boldsymbol{\Lambda}\right)^{+} d \boldsymbol{\Lambda}^{*} V_{j},
\end{aligned}
$$

where $\mathbf{I}$ is the identity matrix of size $M$, and $\left(\lambda_{j} \mathbf{I}-\boldsymbol{\Lambda}\right)^{+}$is the Moore-Penrose inverse of $\lambda_{j} \mathbf{I}-\boldsymbol{\Lambda}$.

Recall the definition of $\lambda_{j}, V_{j}$ and $\hat{\lambda}_{j}, \hat{V}_{j}$, by applying (A.8) and Taylor's expansion,

$$
\begin{aligned}
\hat{\lambda}_{j}-\lambda_{j} & =V_{j}^{\top}\left(\boldsymbol{\Lambda}_{\hat{\mathbf{G}}}-\boldsymbol{\Lambda}\right) V_{j}+o_{p}\left(\frac{1}{\sqrt{n}}\right) \\
& =V_{j}^{\top} \mathbf{E}_{n} V_{j}+o_{p}\left(\frac{1}{\sqrt{n}}\right), \\
\hat{V}_{j}-V_{j} & =\left(\lambda_{j} \mathbf{I}-\boldsymbol{\Lambda}\right)^{+}\left(\boldsymbol{\Lambda}_{\hat{\mathbf{G}}}-\boldsymbol{\Lambda}\right) V_{j}+o_{p}\left(\frac{1}{\sqrt{n}}\right) \\
& =\left(\lambda_{j} \mathbf{I}-\boldsymbol{\Lambda}\right)^{+} \mathbf{E}_{n} V_{j}+o_{p}\left(\frac{1}{\sqrt{n}}\right) .
\end{aligned}
$$

(i) Since we have assumed that $\mathrm{F}(U)=\mathbf{V}_{1}^{* \top} \mathrm{G}(U)$, i.e. $F_{j}(U)=\mathrm{V}_{j}^{\top} \mathrm{G}(U), e_{j}^{\top} \mathbf{F}=\mathrm{V}_{j}^{\top} \mathbf{G}$, $e_{j}^{\top} \mathbf{F}^{\prime \prime}=\mathrm{V}_{j}^{\top} \mathbf{G}^{\prime \prime}$, and $b_{j}=\mathrm{V}_{j}$ by (2.18),

$$
\begin{aligned}
\hat{\lambda}_{j}-\lambda_{j} & =V_{j}^{\top}\left[\left(\frac{1}{2} w_{2}^{K} h^{2}\right) \mathbf{W}_{n}+\mathbf{H}_{n 1}+\mathbf{H}_{n 1}^{\top}+\mathbf{H}_{n 2}\right] V_{j}+o_{p}\left(\frac{1}{\sqrt{n}}\right) \\
& =\left(\frac{1}{2} w_{2}^{K} h^{2}\right) \mathrm{V}_{j}^{\top} \mathbf{W}_{n} \mathrm{~V}_{j}+\mathrm{V}_{j}^{\top}\left(\mathbf{H}_{n 1}+\mathbf{H}_{n 1}^{\top}\right) \mathrm{V}_{j}+\mathrm{V}_{j}^{\top} \mathbf{H}_{n 2} \mathrm{~V}_{j}+o_{p}\left(\frac{1}{\sqrt{n}}\right),
\end{aligned}
$$

with

$$
\left(\frac{1}{2} w_{2}^{K} h^{2}\right) \mathrm{V}_{j}^{\top} \mathbf{W}_{n} \mathrm{~V}_{j}=\left(\frac{1}{2} w_{2}^{K} h^{2}\right)\left[\frac{2}{n} \mathrm{~V}_{j}^{\top} \mathbf{G G}^{\prime \prime \top} \mathrm{V}_{j}-\frac{1}{n} \mathrm{~V}_{j}^{\top} \mathbf{G}\left(\boldsymbol{\varrho}^{\top} \circ \boldsymbol{\sigma}^{\top}\right) \mathrm{V}_{j}\right]
$$




$$
\begin{aligned}
& =\left(\frac{1}{2} w_{2}^{K} h^{2}\right)\left[\frac{2}{n} \sum_{t=1}^{n} F_{j}\left(U_{t}\right) F_{j}^{\prime \prime}\left(U_{t}\right)-\frac{1}{n} \sum_{t=1}^{n} \mathrm{~V}_{j}^{\top}\left(\varrho\left(U_{t}\right) \circ \sigma\left(U_{t}\right)\right) F_{j}\left(U_{t}\right)\right] \\
& =\left(\frac{1}{2} w_{2}^{K} h^{2}\right)\left[\frac{2}{n} \sum_{t=1}^{n} F_{j}\left(U_{t}\right) F_{j}^{\prime \prime}\left(U_{t}\right)-\frac{1}{n} \sum_{t=1}^{n} F_{j}\left(U_{t}\right) b_{j}^{\top}\left(\varrho\left(U_{t}\right) \circ \sigma\left(U_{t}\right)\right)\right] \\
\mathrm{V}_{j}^{\top}\left(\mathbf{H}_{n 1}+\mathbf{H}_{n 1}^{\top}\right) \mathrm{V}_{j} & =\frac{2}{n} \mathrm{~V}_{j}^{\top} \mathbf{G} \boldsymbol{\epsilon}^{\top} \mathrm{V}_{j}-\frac{1}{n} \mathrm{~V}_{j}^{\top} \mathbf{G}\left(\varrho^{\top} \circ \boldsymbol{\xi}^{\top}\right) \mathrm{V}_{j} \\
& =\frac{2}{n} \sum_{t=1}^{n} F_{j}\left(U_{t}\right) \mathrm{V}_{j}^{\top} \tilde{\epsilon}_{t}-\frac{1}{n} \sum_{t=1}^{n} F_{j}\left(U_{t}\right) \mathrm{V}_{j}^{\top}\left(\varrho\left(U_{t}\right) \circ \tilde{\xi}_{t}\right) \\
& =\frac{1}{n} \sum_{t=1}^{n} F_{j}\left(U_{t}\right) b_{j}^{\top}\left[2 \tilde{\epsilon}_{t}-\left(\varrho\left(U_{t}\right) \circ \tilde{\xi}_{t}\right)\right] \\
\mathrm{V}_{j}^{\top} \mathbf{H}_{n 2} \mathrm{~V}_{j} & =\mathrm{V}_{j}^{\top}\left[\frac{1}{n} \mathbf{G G}^{\top}-E\left\{G(U) G(U)^{\top}\right\}\right] \mathrm{V}_{j}=\frac{1}{n} \sum_{t=1}^{n} F_{j}^{2}\left(U_{t}\right)-E F_{j}^{2}(U),
\end{aligned}
$$

where $\boldsymbol{\epsilon}=\left(\tilde{\epsilon}_{1}, \ldots, \tilde{\epsilon}_{n}\right)$, and $\boldsymbol{\xi}=\left(\tilde{\xi}_{1}, \ldots, \tilde{\xi}_{n}\right)$.

Then, because $F_{j}(\cdot), F_{j}^{\prime \prime}(\cdot)$ are uniformly continuous by $(\mathrm{C} 4)$, together with (C1) and $(\mathrm{C} 2)$, we could show that $E\left|F_{j}\left(U_{t}\right)\right|^{4+\delta}<\infty, E\left|F_{j}\left(U_{t}\right) F_{j}^{\prime \prime}\left(U_{t}\right)\right|^{2+\delta}<\infty, E \mid b_{j}^{\top}\left(\varrho\left(U_{t}\right) \circ\right.$ $\left.\sigma\left(U_{t}\right)\right)\left.F_{j}\left(U_{t}\right)\right|^{2+\delta}<\infty, E\left|b_{j}^{\top} \tilde{\epsilon}_{t} F_{j}\left(U_{t}\right)\right|^{2+\delta}<\infty, E\left|b_{j}^{\top}\left(\varrho\left(U_{t}\right) \circ \tilde{\xi}_{t}\right) F_{j}\left(U_{t}\right)\right|^{2+\delta}<\infty$, and by Hölder's inequality, $E\left|2 b_{j}^{\top} \tilde{\epsilon}_{t} F_{j}\left(U_{t}\right)-b_{j}^{\top}\left(\varrho\left(U_{t}\right) \circ \tilde{\xi}_{t}\right) F_{j}\left(U_{t}\right)+F_{j}^{2}\left(U_{t}\right)\right|^{2+\delta}<\infty$ could be obtained accordingly.

Under the $\alpha$-mixing condition (C3), $\sum_{N=1}^{\infty} \alpha(N)^{\frac{\delta}{2+\delta}} \leq \sum_{N=1}^{\infty} N^{-\left(2+\frac{2}{\delta}\right)\left(\frac{\delta}{2+\delta}\right)}=\sum_{N=1}^{\infty}$ $N^{-2+\frac{2}{2+\delta}}<\infty$, then

$$
\begin{aligned}
\frac{1}{n} \sum_{t=1}^{n} F_{j}\left(U_{t}\right) F_{j}^{\prime \prime}\left(U_{t}\right) & =E\left\{F_{j}(U) F^{\prime \prime}(U)\right\}+O\left(\frac{1}{\sqrt{n}}\right), \\
\frac{1}{n} \sum_{t=1}^{n} F_{j}\left(U_{t}\right) b_{j}^{\top}\left(\varrho\left(U_{t}\right) \circ \sigma\left(U_{t}\right)\right) & =E\left\{F_{j}(U) b_{j}^{\top}(\varrho(U) \circ \sigma(U))\right\}+O\left(\frac{1}{\sqrt{n}}\right),
\end{aligned}
$$

thus

$$
\begin{aligned}
& \sqrt{n}\left(\hat{\lambda}_{j}-\lambda_{j}-\left(\frac{1}{2} w_{2}^{K} h^{2}\right) E\left\{2 F_{j}(U) F^{\prime \prime}(U)-b_{j}^{\top} F_{j}(U)(\varrho(U) \circ \sigma(U))\right\}\right) \\
= & \frac{1}{\sqrt{n}} \sum_{t=1}^{n}\left[2 b_{j}^{\top} \tilde{\epsilon}_{t} F_{j}\left(U_{t}\right)-b_{j}^{\top}\left(\varrho\left(U_{t}\right) \circ \tilde{\xi}_{t}\right) F_{j}\left(U_{t}\right)+F_{j}^{2}\left(U_{t}\right)-E F_{j}^{2}(U)\right]+o(1) .
\end{aligned}
$$

Let $I_{j, t}=2 b_{j}^{\top} \tilde{\epsilon}_{t} F_{j}\left(U_{t}\right)-b_{j}^{\top}\left(\varrho\left(U_{t}\right) \circ \tilde{\xi}_{t}\right) F_{j}\left(U_{t}\right)+F_{j}^{2}\left(U_{t}\right)-E F_{j}^{2}(U)$, since $E\left\{\tilde{\epsilon}_{t} \mid U_{t}\right\}=0$, $E\left\{\tilde{\xi}_{t} \mid U_{t}\right\}=0$, for a particular $t$,

$$
\operatorname{Var}\left(I_{j, t}\right)=\operatorname{Var}\left\{2 b_{j}^{\top} \tilde{\epsilon}_{t} F_{j}\left(U_{t}\right)-b_{j}^{\top}\left(\varrho\left(U_{t}\right) \circ \tilde{\xi}_{t}\right) F_{j}\left(U_{t}\right)\right\}+\operatorname{Var}\left\{F_{j}^{2}\left(U_{t}\right)-E F_{j}^{2}(U)\right\}
$$




$$
=\operatorname{Var}\left\{b_{j}^{\top}\left[2 \tilde{\epsilon}_{t}-\left(\varrho\left(U_{t}\right) \circ \tilde{\xi}_{t}\right)\right] F_{j}\left(U_{t}\right)\right\}+E\left\{F_{j}^{4}\left(U_{t}\right)\right\}-\lambda_{j}^{2},
$$

for time $t$ and $t+s$,

$$
\begin{aligned}
\operatorname{Cov}\left(I_{j, t}, I_{j, t+s}\right)= & \operatorname{Cov}\left(b_{j}^{\top}\left[2 \tilde{\epsilon}_{t}-\left(\varrho\left(U_{t}\right) \circ \tilde{\xi}_{t}\right)\right] F_{j}\left(U_{t}\right)+F_{j}^{2}\left(U_{t}\right)-E F_{j}^{2}(U), b_{j}^{\top}\left[2 \tilde{\epsilon}_{t+s}-\right.\right. \\
& \left.\left.\left(\varrho\left(U_{t+s}\right) \circ \tilde{\xi}_{t+s}\right)\right] F_{j}\left(U_{t+s}\right)+F_{j}^{2}\left(U_{t+s}\right)-E F_{j}^{2}(U)\right) \\
= & E\left\{b_{j}^{\top}\left[2 \tilde{\epsilon}_{t}-\left(\varrho\left(U_{t}\right) \circ \tilde{\xi}_{t}\right)\right]\left[2 \tilde{\epsilon}_{t+s}^{\top}-\left(\varrho^{\top}\left(U_{t+s}\right) \circ \tilde{\xi}_{t+s}^{\top}\right)\right] b_{j} F_{j}\left(U_{t}\right) F_{j}\left(U_{t+s}\right)\right\} \\
& +E\left\{F_{j}^{2}\left(U_{t}\right) F_{j}^{2}\left(U_{t+s}\right)\right\} .
\end{aligned}
$$

hence, by CLT result for $\alpha$-mixing series,

$$
\sqrt{n}\left(\hat{\lambda}_{j}-\lambda_{j}-\left(\frac{1}{2} w_{2}^{K} h^{2}\right) E\left\{2 F_{j}(U) F^{\prime \prime}(U)-b_{j}^{\top} F_{j}(U)(\varrho(U) \circ \sigma(U))\right\}\right) \stackrel{d}{\longrightarrow} N\left(0, \sigma_{\lambda_{j}}^{2}\right),
$$

where

$$
\begin{aligned}
\sigma_{\lambda_{j}}^{2}= & E\left\{I_{j, 1}^{2}\right\}+2 \sum_{s=1}^{\infty} E\left\{I_{j, 1}, I_{j, s+1}\right\} \\
= & E\left\{F_{j}^{2}\left(U_{1}\right) b_{j}^{\top} \operatorname{Cov}\left(2 \tilde{\epsilon}_{1}-\varrho\left(U_{1}\right) \circ \tilde{\xi}_{1} \mid U_{1}\right) b_{j}\right\}+E\left\{F_{j}^{4}\left(U_{1}\right)\right\}-\lambda_{j}^{2} \\
& +2 \sum_{s=1}^{\infty} E\left\{F_{j}\left(U_{1}\right) F_{j}\left(U_{s+1}\right) b_{j}^{\top} \operatorname{Cov}\left(2 \tilde{\epsilon}_{1}-\varrho\left(U_{1}\right) \circ \tilde{\xi}_{1}, 2 \tilde{\epsilon}_{s+1}-\varrho\left(U_{s+1}\right) \circ \tilde{\xi}_{s+1} \mid U_{1}, U_{s+1}\right) b_{j}\right\} \\
& +2 \sum_{s=1}^{\infty} E\left\{\left(F_{j}^{2}\left(U_{1}\right)-\lambda_{j}\right)\left(F_{j}^{2}\left(U_{s+1}\right)-\lambda_{j}\right)\right\}
\end{aligned}
$$

(ii) Similarly, consider the asymptotic properties of the estimated eigenvector $\hat{\mathrm{V}}_{j}$. Let $\mathbf{I}_{p}$ be the identity matrix of size $p$, then substitute (A.7) into (A.10),

$$
\begin{aligned}
\hat{\mathrm{V}}_{j}-\mathrm{V}_{j} & =\left(\lambda_{j} \mathbf{I}-\boldsymbol{\Lambda}\right)^{+} \mathbf{E}_{n} \mathrm{~V}_{j}+o_{p}\left(\frac{1}{\sqrt{n}}\right) \\
& =\left(\lambda_{j} \mathbf{I}-\boldsymbol{\Lambda}\right)^{+}\left[\left(\frac{1}{2} w_{2}^{K} h^{2}\right) \mathbf{W}_{n}+\mathbf{H}_{n 1}+\mathbf{H}_{n 1}^{\top}+\mathbf{H}_{n 2}\right] \mathrm{V}_{j}+o_{p}\left(\frac{1}{\sqrt{n}}\right) .
\end{aligned}
$$

Specifically, $\mathbf{G}=\mathbf{B F}=\mathbf{V}_{1}^{*} \mathbf{F}$ by (2.18), and $\sum_{i=1}^{p} \mathrm{~V}_{i} \mathrm{~V}_{i}^{\top}=\mathbf{V}_{1}^{*} \mathbf{V}_{1}^{* \top}, \mathbf{V}_{1}^{* \top} \mathbf{V}_{1}^{*}=\mathbf{I}_{p}$. Moreover, $\left(\lambda_{j} \mathbf{I}-\boldsymbol{\Lambda}\right)^{\top} \boldsymbol{\Lambda} \mathrm{V}_{j}=\left(\lambda_{j} \mathbf{I}-\boldsymbol{\Lambda}\right) \lambda_{j} \mathrm{~V}_{j}=0$, which means that $\left(\lambda_{j} \mathbf{I}-\boldsymbol{\Lambda}\right)^{+} \boldsymbol{\Lambda} \mathrm{V}_{j}=0$. Thus,

$$
\begin{aligned}
& \left(\frac{1}{2} w_{2}^{K} h^{2}\right)\left(\lambda_{j} \mathbf{I}-\boldsymbol{\Lambda}\right)^{+} \mathbf{W}_{n} \mathrm{~V}_{j} \\
& \quad=\left(\frac{1}{2} w_{2}^{K} h^{2}\right)\left(\lambda_{j} \mathbf{I}-\boldsymbol{\Lambda}\right)^{+} \frac{1}{n} \sum_{t=1}^{n}\left[\sum_{i=1}^{p} \mathrm{~V}_{i} F_{i}\left(U_{t}\right) F_{j}^{\prime \prime}\left(U_{t}\right)+\sum_{i=1}^{p} \mathrm{~V}_{i} F_{j}\left(U_{t}\right) F_{i}^{\prime \prime}\left(U_{t}\right)\right.
\end{aligned}
$$




$$
\begin{gathered}
\left.-\frac{1}{2} \sum_{i=1}^{p} \mathrm{~V}_{i} F_{i}\left(U_{t}\right) \mathrm{V}_{j}^{\top}\left(\varrho\left(U_{t}\right) \circ \sigma\left(U_{t}\right)\right)-\frac{1}{2} F_{j}\left(U_{t}\right)\left(\varrho\left(U_{t}\right) \circ \sigma\left(U_{t}\right)\right)\right] \\
\left(\lambda_{j} \mathbf{I}-\boldsymbol{\Lambda}\right)^{+} \mathbf{H}_{n 1} \mathrm{~V}_{j}=\left(\lambda_{j} \mathbf{I}-\boldsymbol{\Lambda}\right)^{+} \frac{1}{n} \sum_{t=1}^{n} \sum_{i=1}^{p} \mathrm{~V}_{i} \mathrm{~V}_{j}^{\top}\left[\tilde{\epsilon}_{t}-\frac{1}{2}\left(\varrho\left(U_{t}\right) \circ \tilde{\xi}_{t}\right)\right] F_{i}\left(U_{t}\right), \\
\left(\lambda_{j} \mathbf{I}-\boldsymbol{\Lambda}\right)^{+} \mathbf{H}_{n 1}^{\top} \mathrm{V}_{j}=\left(\lambda_{j} \mathbf{I}-\boldsymbol{\Lambda}\right)^{+} \frac{1}{n} \sum_{t=1}^{n}\left[\tilde{\epsilon}_{t}-\frac{1}{2}\left(\varrho\left(U_{t}\right) \circ \tilde{\xi}_{t}\right)\right] F_{j}\left(U_{t}\right), \\
\left(\lambda_{j} \mathbf{I}-\boldsymbol{\Lambda}\right)^{+} \mathbf{H}_{n 2} \mathrm{~V}_{j}=\left(\lambda_{j} \mathbf{I}-\boldsymbol{\Lambda}\right)^{+}\left[\frac{1}{n} \mathbf{G G}^{\top}-\boldsymbol{\Lambda}\right] \mathrm{V}_{j}=\left(\lambda_{j} \mathbf{I}-\boldsymbol{\Lambda}\right)^{+} \frac{1}{n} \sum_{t=1}^{n} \sum_{i=1}^{p} \mathrm{~V}_{i} F_{i}\left(U_{t}\right) F_{j}\left(U_{t}\right) .
\end{gathered}
$$

To investigate the asymptotic normality of the eigenvector $\hat{\mathrm{V}}_{j}$, we consider the asymptotic result of $\mathrm{y}^{\top} \hat{\mathrm{V}}_{j}$ for $\mathrm{y} \in \mathbb{R}^{M}$. Under the $\alpha$-mixing condition $(\mathrm{C} 3), \sum_{N=1}^{\infty} \alpha(N)^{\frac{\delta}{2+\delta}} \leq$ $\sum_{N=1}^{\infty} N^{-\left(2+\frac{2}{\delta}\right)\left(\frac{\delta}{2+\delta}\right)}=\sum_{N=1}^{\infty} N^{-2+\frac{2}{2+\delta}}<\infty$. Let

$$
\begin{aligned}
\mathrm{W}_{j, t}= & \left(\lambda_{j} \mathbf{I}-\boldsymbol{\Lambda}\right)^{+}\left[\sum_{i=1}^{p} \mathrm{~V}_{i} F_{i}\left(U_{t}\right) F_{j}^{\prime \prime}\left(U_{t}\right)+\sum_{i=1}^{p} \mathrm{~V}_{i} F_{j}\left(U_{t}\right) F_{i}^{\prime \prime}\left(U_{t}\right)\right. \\
& \left.-\frac{1}{2} \sum_{i=1}^{p} \mathrm{~V}_{i} F_{i}\left(U_{t}\right) \mathrm{V}_{j}^{\top}\left(\varrho\left(U_{t}\right) \circ \sigma\left(U_{t}\right)\right)-\frac{1}{2} F_{j}\left(U_{t}\right)\left(\varrho\left(U_{t}\right) \circ \sigma\left(U_{t}\right)\right)\right] \\
\mathrm{H}_{j, t}= & \left(\lambda_{j} \mathbf{I}-\boldsymbol{\Lambda}\right)^{+}\left[\sum_{i=1}^{p} \mathrm{~V}_{i} \mathrm{~V}_{j}^{\top}\left(\tilde{\epsilon}_{t}-\frac{1}{2}\left(\varrho\left(U_{t}\right) \circ \tilde{\xi}_{t}\right)\right) F_{i}\left(U_{t}\right)+\left(\tilde{\epsilon}_{t}-\frac{1}{2}\left(\varrho\left(U_{t}\right) \circ \tilde{\xi}_{t}\right)\right) F_{j}\left(U_{t}\right)\right. \\
& \left.+\sum_{i=1}^{p} \mathrm{~V}_{i} F_{i}\left(U_{t}\right) F_{j}\left(U_{t}\right)\right] \\
= & \left(\lambda_{j} \mathbf{I}-\boldsymbol{\Lambda}\right)^{+}\left[\left(\sum_{i=1}^{p} \mathrm{~V}_{i} \mathrm{~V}_{j}^{\top} F_{i}\left(U_{t}\right)+F_{j}\left(U_{t}\right) \mathbf{I}\right)\left(\tilde{\epsilon}_{t}-\frac{1}{2} \varrho\left(U_{t}\right) \circ \tilde{\xi}_{t}\right)+\sum_{i=1}^{p} \mathrm{~V}_{i} F_{i}\left(U_{t}\right) F_{j}\left(U_{t}\right)\right]
\end{aligned}
$$

by (C1), (C2) and (C4), for the same $\delta$ in the assumptions, $E\left|\mathrm{y}^{\top} \mathrm{H}_{j, t}\right|^{2+\delta}<\infty$, and $E\left|\mathrm{y}^{\top} \mathrm{W}_{j, t}\right|^{2+\delta}<\infty$, then for an arbitrary linear combination $\mathrm{y}^{\top} \hat{\mathrm{V}}_{j}$ for $y \in \mathbb{R}^{M}$,

$$
\sqrt{n}\left(\mathrm{y}^{\top} \hat{\mathrm{V}}_{j}-\mathrm{y}^{\top} \mathrm{V}_{j}-\left(\frac{1}{2} w_{2}^{K} h^{2}\right) E \mathrm{y}^{\top} \mathrm{W}_{j, 1}\right)=\frac{1}{n} \sum_{t=1}^{n} \mathrm{y}^{\top} \mathrm{H}_{j, t}+o_{p}(1),
$$

hence, by CLT result for $\alpha$-mixing series, which means that

$$
\sqrt{n}\left(\mathrm{y}^{\top} \hat{\mathrm{V}}_{j}-\mathrm{y}^{\top} \mathrm{V}_{j}-\left(\frac{1}{2} w_{2}^{K} h^{2}\right) E \mathrm{y}^{\top} \mathrm{W}_{j, 1}\right) \stackrel{d}{\longrightarrow} N\left(0, \mathrm{y}^{\top} \boldsymbol{\Sigma}_{\mathrm{V}_{j}} \mathrm{y}\right)
$$

where

$$
\begin{aligned}
E \mathrm{~W}_{j, 1}=E\left\{( \lambda _ { j } \mathbf { I } - \boldsymbol { \Lambda } ) ^ { + } \left[\sum_{i=1}^{p} \mathrm{~V}_{i} F_{i}\left(U_{t}\right)\right.\right. & F_{j}^{\prime \prime}\left(U_{t}\right)+\sum_{i=1}^{p} \mathrm{~V}_{i} F_{j}\left(U_{t}\right) F_{i}^{\prime \prime}\left(U_{t}\right) \\
& \left.\left.-\frac{1}{2} \sum_{i=1}^{p} \mathrm{~V}_{i} F_{i}\left(U_{t}\right) \mathrm{V}_{j}^{\top}\left(\varrho\left(U_{t}\right) \circ \sigma\left(U_{t}\right)\right)-\frac{1}{2} F_{j}\left(U_{t}\right)\left(\varrho\left(U_{t}\right) \circ \sigma\left(U_{t}\right)\right)\right]\right\},
\end{aligned}
$$




$$
\begin{aligned}
\boldsymbol{\Sigma}_{\mathrm{V}_{j}}= & \operatorname{Cov}\left(\mathrm{H}_{j, 1}\right)+2 \sum_{s=1}^{\infty} \operatorname{Cov}\left(\mathrm{H}_{j, 1}, \mathrm{H}_{j, s+1}\right) \\
= & \left(\lambda_{j} \mathbf{I}-\boldsymbol{\Lambda}\right)^{+}\left[\left(\sum_{i=1}^{p} \mathrm{~V}_{i} \mathrm{~V}_{j}^{\top} F_{i}\left(U_{1}\right)+F_{j}\left(U_{1}\right) \mathbf{I}\right) \operatorname{Cov}\left(\tilde{\epsilon}_{1}-\frac{1}{2} \varrho\left(U_{1}\right) \circ \tilde{\xi}_{1} \mid U_{1}\right)\left(\sum_{i=1}^{p} \mathrm{~V}_{j} \mathrm{~V}_{i}^{\top} F_{i}\left(U_{1}\right)+F_{j}\left(U_{1}\right) \mathbf{I}\right)\right. \\
& +2 \sum_{s=1}^{\infty} \operatorname{Cov}\left(\left(\sum_{i=1}^{p} \mathrm{~V}_{i} \mathrm{~V}_{j}^{\top} F_{i}\left(U_{1}\right)+F_{j}\left(U_{1}\right) \mathbf{I}\right)\left(\tilde{\epsilon}_{1}-\frac{1}{2} \varrho\left(U_{1}\right) \circ \tilde{\xi}_{1}\right),\left(\sum_{i=1}^{p} \mathrm{~V}_{i} \mathrm{~V}_{j}^{\top} F_{i}\left(U_{s+1}\right)+F_{j}\left(U_{s+1}\right) \mathbf{I}\right)\right. \\
& \left.\left(\tilde{\epsilon}_{s+1}-\frac{1}{2} \varrho\left(U_{s+1}\right) \circ \tilde{\xi}_{s+1}\right)\right)+\left(\sum_{i=1}^{p} \mathrm{~V}_{i} F_{i}\left(U_{1}\right) F_{j}\left(U_{1}\right)\right)\left(\sum_{i=1}^{p} \mathrm{~V}_{i}^{\top} F_{i}\left(U_{1}\right) F_{j}\left(U_{1}\right)\right) \\
& \left.+2 \sum_{s=1}^{\infty} \operatorname{Cov}\left(\sum_{i=1}^{p} \mathrm{~V}_{i} F_{i}\left(U_{1}\right) F_{j}\left(U_{1}\right), \sum_{i=1}^{p} \mathrm{~V}_{i} F_{i}\left(U_{s+1}\right) F_{j}\left(U_{s+1}\right)\right)\right]\left(\lambda_{j} \mathbf{I}-\boldsymbol{\Lambda}\right)^{+},
\end{aligned}
$$

therefore, by cramér-wold theorem,

$$
\sqrt{n}\left(\hat{\mathrm{V}}_{j}-\mathrm{V}_{j}-\left(\frac{1}{2} w_{2}^{K} h^{2}\right) E \mathrm{~W}_{j, 1}\right) \stackrel{d}{\longrightarrow} \mathbf{N}_{M}\left(0, \boldsymbol{\Sigma}_{\mathrm{V}_{j}}\right)
$$

Proof of Theorem 3.3. From (A.6), we could directly have the following equation,

$$
\begin{aligned}
\hat{\mathrm{G}}(u)= & \mathrm{G}(u)+\frac{1}{2} w_{2}^{K} h^{2}\left[\varrho^{\prime \prime}(u)-\frac{1}{2} \varrho(u) \circ \sigma(u)\right]+\frac{1}{n} \boldsymbol{\epsilon} K_{f}(u)-\frac{1}{2 n} \varrho(u) \circ\left(\boldsymbol{\sigma}_{\xi}(u) K * K_{f}(u)\right) \\
& -\frac{1}{2} w_{2}^{K} h^{2}\left[\frac{1}{n} \varrho^{\prime \prime} 1_{n}-\frac{1}{2 n}(\varrho \circ \boldsymbol{\sigma}) 1_{n}\right]-\frac{1}{n} \mathbf{G} 1_{n}-\left[\frac{1}{n^{2}} \boldsymbol{\epsilon} \mathbf{K}_{f} 1_{n}-\frac{1}{2 n^{2}}\left(\varrho \circ\left(\boldsymbol{\sigma}_{\xi} \mathbf{K} * \mathbf{K}_{f}\right)\right) 1_{n}\right] \\
& +\delta_{n},
\end{aligned}
$$

and recall (2.18), (A.7), $\varrho^{\prime \prime}(u)=\mathbf{B} F^{\prime \prime}(u)=\mathbf{V}_{1}^{*} \mathrm{~F}^{\prime \prime}(u), \varrho^{\prime \prime}=\mathbf{B F}^{\prime \prime}=\mathbf{V}_{1}^{*} \mathbf{F}$, hence,

$$
\begin{aligned}
& \hat{F}_{j}(u)-F_{j}(u) \\
= & \hat{\mathrm{V}}_{j}^{\top} \hat{\mathrm{G}}(u)-\mathrm{V}_{j}^{\top} \mathrm{G}(u)=\left(\mathrm{V}_{j}+\left(\lambda_{j} \mathbf{I}-\boldsymbol{\Lambda}\right)^{+} \mathbf{E}_{n} \mathrm{~V}_{j}\right)^{\top}(\mathrm{G}(u)+\hat{\mathrm{G}}(u)-\mathrm{G}(u))-\mathrm{V}_{j}^{\top} \mathrm{G}(u) \\
= & \mathrm{V}_{j}^{\top}\left(\frac{1}{2} w_{2}^{K} h^{2}\right) \mathbf{W}_{n}\left(\lambda_{j} \mathbf{I}-\boldsymbol{\Lambda}\right)^{+} \mathrm{G}(u)+\mathrm{V}_{j}^{\top}(\hat{\mathrm{G}}(u)-\mathrm{G}(u))+\delta_{n} \\
= & \mathrm{V}_{j}^{\top}\left(\frac{1}{2} w_{2}^{K} h^{2}\right) \mathbf{W}_{n}\left(\lambda_{j} \mathbf{I}-\boldsymbol{\Lambda}\right)^{+} \mathbf{V}_{1}^{*} \mathrm{~F}(u)+\left(\frac{1}{2} w_{2}^{K} h^{2}\right)\left[F_{j}^{\prime \prime}(u)-\frac{1}{2} \mathrm{~V}_{j}^{\top}(\varrho(u) \circ \sigma(u))-\left(\frac{1}{n} e_{j}^{\top} \mathbf{F}^{\prime \prime} 1_{n}\right.\right. \\
& \left.\left.-\frac{1}{2 n} \mathrm{~V}_{j}^{\top}(\varrho \circ \boldsymbol{\sigma}) 1_{n}\right)\right]+\frac{1}{n} \mathrm{~V}_{j}^{\top}\left[\boldsymbol{\epsilon} K_{f}(u)-\frac{1}{2} \varrho(u) \circ\left(\boldsymbol{\sigma}_{\xi}(u) K * K_{f}(u)\right)\right]+\delta_{n} \\
= & \left(\frac{1}{2} w_{2}^{K} h^{2}\right) A_{1}(u)+A_{2}(u)+\delta_{n},
\end{aligned}
$$

where

$A_{1}(u)=V_{j}^{\top} \mathbf{W}_{n}\left(\lambda_{j} \mathbf{I}-\boldsymbol{\Lambda}\right)^{+} \mathbf{V}_{1}^{*} \mathrm{~F}(u)+F_{j}^{\prime \prime}(u)-\frac{1}{2} \mathrm{~V}_{j}^{\top}(\varrho(u) \circ \sigma(u))-\left(\frac{1}{n} e_{j}^{\top} \mathbf{F}^{\prime \prime} 1_{n}-\frac{1}{2 n} \mathrm{~V}_{j}^{\top}(\varrho \circ \boldsymbol{\sigma}) 1_{n}\right)$ 


$$
\begin{aligned}
= & \frac{1}{n} \sum_{t=1}^{n}\left[\sum_{i=1}^{p} F_{i}\left(U_{t}\right) F_{j}^{\prime \prime}\left(U_{t}\right) \mathrm{V}_{i}^{\top}+\sum_{i=1}^{p} F_{j}\left(U_{t}\right) F_{i}^{\prime \prime}\left(U_{t}\right) \mathrm{V}_{i}^{\top}-\frac{1}{2} \sum_{i=1}^{p} \mathrm{~V}_{j}^{\top}\left(\varrho\left(U_{t}\right) \circ \sigma\left(U_{t}\right)\right) F_{i}\left(U_{t}\right) \mathrm{V}_{i}^{\top}\right. \\
& \left.-\frac{1}{2}\left(\varrho^{\top}\left(U_{t}\right) \circ \sigma^{\top}\left(U_{t}\right)\right) F_{j}\left(U_{t}\right)\right]\left(\lambda_{j} \mathbf{I}-\boldsymbol{\Lambda}\right)^{+} \mathbf{V}_{1}^{*} \mathrm{~F}(u)+\left[F_{j}^{\prime \prime}(u)-\frac{1}{2} \mathrm{~V}_{j}^{\top}(\varrho(u) \circ \sigma(u))\right. \\
& \left.-\left(\frac{1}{n} \sum_{t=1}^{n} F_{j}^{\prime \prime}\left(U_{t}\right)-\frac{1}{2 n} \sum_{t=1}^{n} \mathrm{~V}_{j}^{\top}\left(\varrho\left(U_{t}\right) \circ \sigma\left(U_{t}\right)\right)\right)\right] \\
A_{2}(u)= & \frac{1}{n} \mathrm{~V}_{j}^{\top}\left[\epsilon K_{f}(u)-\frac{1}{2} \varrho(u) \circ\left(\sigma_{\xi}(u) K * K_{f}(u)\right)\right] \\
= & \frac{1}{n f_{U}(u)} \sum_{t=1}^{n}\left[V_{j}^{\top} \tilde{\epsilon}_{t} K_{h}\left(U_{t}-u\right)-\frac{1}{2} \mathrm{~V}_{j}^{\top}\left(\varrho(u) \circ \tilde{\sigma}_{\xi, t}(u)\right) K * K_{h}\left(U_{t}-u\right)\right] .
\end{aligned}
$$

Then

$$
\begin{aligned}
E A_{1}(u)= & {\left[F_{j}^{\prime \prime}(u)-E F_{j}^{\prime \prime}(U)\right]-\frac{1}{2} \mathrm{~V}_{j}^{\top}[(\varrho(u) \circ \sigma(u))-E(\varrho(U) \circ \sigma(U))] } \\
& +E\left[F_{i}(U) F_{j}^{\prime \prime}(U) \mathrm{V}_{i}^{\top}+F_{j}(U) F_{i}^{\prime \prime}(U) \mathrm{V}_{i}^{\top}-\frac{1}{2} \mathrm{~V}_{j}^{\top}(\varrho(U) \circ \sigma(U)) F_{i}(U) \mathrm{V}_{i}^{\top}\right. \\
& \left.-\frac{1}{2}\left(\varrho^{\top}(U) \circ \sigma^{\top}(U)\right) F_{j}(U)\right]\left(\lambda_{j} \mathbf{I}-\boldsymbol{\Lambda}\right)^{+} \mathbf{V}_{1}^{*} \mathrm{~F}(u), \\
E A_{2}(u)= & \frac{1}{n f_{U}(u)}\left[V_{j}^{\top} \tilde{\epsilon}_{1} K_{h}\left(U_{1}-u\right)-\frac{1}{2} \mathrm{~V}_{j}^{\top}\left(\varrho(u) \circ \tilde{\sigma}_{\xi, 1}(u)\right) K * K_{h}\left(U_{1}-u\right)\right]=0,
\end{aligned}
$$

and let

$$
A_{2}(u)=\frac{1}{n} \sum_{t=1}^{n} R_{t}(u)
$$

with

$$
R_{t}(u)=\frac{1}{f_{U}(u)}\left[V_{j}^{\top} \tilde{\epsilon}_{t} K_{h}\left(U_{t}-u\right)-\frac{1}{2} \mathrm{~V}_{j}^{\top}\left(\varrho(u) \circ \tilde{\sigma}_{\xi, t}(u)\right) K * K_{h}\left(U_{t}-u\right)\right] .
$$

Note that

$$
\begin{aligned}
\operatorname{Var}\left(R_{1}(u)\right)= & \frac{1}{h f_{U}(u)} V_{j}^{\top}\left[\nu_{K}^{2} \operatorname{Var}\left(\tilde{\epsilon}_{1}\right)+\frac{1}{4} \nu_{K * K}^{2}\left(\left(\varrho(u) \varrho^{\top}(u)\right) \circ \operatorname{Var}\left(\tilde{\xi}_{1}\right)\right)\right. \\
& \left.-\nu_{K, K * K} E\left\{\tilde{\epsilon}_{1}\left(\varrho^{\top}(u) \circ \tilde{\xi}_{1}^{\top}\right)\right\}\right] V_{j}+o\left(\frac{1}{h}\right)
\end{aligned}
$$

by stationarity in (C3), we have

$$
\operatorname{Var}\left(A_{2}(u)\right)=\frac{1}{n} \operatorname{Var}\left(R_{1}(u)\right)+\frac{2}{n} \sum_{s=1}^{n-1}\left(1-\frac{s}{n}\right) \operatorname{Cov}\left(R_{1}(u), R_{s+1}(u)\right) .
$$

Let $d_{n} \rightarrow \infty$ be a sequence of integers such that $h d_{n} \rightarrow 0$. Define

$$
Q_{1}=\sum_{s=1}^{d_{n}-1}\left|\operatorname{Cov}\left(R_{1}(u), R_{s+1}(u)\right)\right|, \quad Q_{2}=\sum_{s=d_{n}}^{n-1}\left|\operatorname{Cov}\left(R_{1}(u), R_{s+1}(u)\right)\right| .
$$


18

Conditioning on $\left(U_{1}, U_{s+1}\right)$, and by (C2), (C4) and (C5),

$$
\begin{aligned}
& \left|\operatorname{Cov}\left(R_{1}(u), R_{s+1}(u)\right)\right| \\
= & \left|E\left\{E\left(R_{1}(u), R_{s+1}(u) \mid U_{1}, U_{s+1}\right)\right\}\right| \\
= & \mid E\left\{E \left(\frac{1}{f^{2}(u)}\left[V_{j}^{\top} \tilde{\epsilon}_{1} K_{h}\left(U_{1}-u\right)-\frac{1}{2} \mathrm{~V}_{j}^{\top}\left(\varrho(u) \circ \tilde{\sigma}_{\xi, 1}(u)\right) K * K_{h}\left(U_{1}-u\right)\right]\right.\right. \\
& {\left.\left.\left[V_{j}^{\top} \tilde{\epsilon}_{t} K_{h}\left(U_{s+1}-u\right)-\frac{1}{2} \mathrm{~V}_{j}^{\top}\left(\varrho(u) \circ \tilde{\sigma}_{\xi, s+1}(u)\right) K * K_{h}\left(U_{s+1}-u\right)\right]\right) \mid U_{1}, U_{s+1}\right\} \mid } \\
\leq & C L_{2} \leq M_{0}
\end{aligned}
$$

for $M_{0}>0$ and some generic constant $C>0$. Then it follows that $Q_{1} \leq d_{n} M_{0}$. We now consider the contribution of $Q_{2}$. For this $\alpha$-mixing process, by Davydov's lemma,

$$
\left|\operatorname{Cov}\left(R_{1}(u), R_{s+1}(u)\right)\right|=E\left|R_{1}(u) R_{s+1}(u)\right| \leq 8[\alpha(s)]^{\frac{\delta}{1+\delta}}\left\{E\left|R_{1}\right|^{2(1+\delta)}\right\}^{\frac{1}{1+\delta}} .
$$

By conditioning on $U_{1}$, and using (C2) and (C3),

$E\left|R_{1}\right|^{2(1+\delta)}=E\left|\frac{V_{j}^{\top} \tilde{\epsilon}_{1} K_{h}\left(U_{1}-u\right)-\frac{1}{2} \mathrm{~V}_{j}^{\top}\left(\varrho(u) \circ \tilde{\sigma}_{\xi, 1}(u)\right) K * K_{h}\left(U_{1}-u\right)}{f_{U}(u)}\right|^{2(1+\delta)} \leq C L_{2} h^{-2(1+\delta)+1}$.

Hence, for $\frac{\delta}{1+\delta}<\gamma<1$,

$$
\begin{aligned}
Q_{2} & \leq \sum_{s=d_{n}}^{n-1} 8[\alpha(s)]^{\frac{\delta}{1+\delta}}\left\{E\left|R_{1}\right|^{2(1+\delta)}\right\}^{\frac{1}{1+\delta}} \leq\left(C L_{2}\right)^{\frac{1}{1+\delta}} 8\left(h^{-2(1+\delta)+1}\right)^{\frac{1}{1+\delta}} \sum_{s=d_{n}}^{\infty}\left[s^{-\beta}\right]^{\frac{\delta}{1+\delta}} \\
& \leq M_{1} h^{-2+\frac{1}{1+\delta}} \sum_{s=d_{n}}^{\infty} s^{-2}=M_{1} h^{-2+\frac{1}{1+\delta}} d_{n}^{-\gamma} \sum_{s=d_{n}}^{\infty} s^{-2+\gamma}=o(1 / h)
\end{aligned}
$$

by taking $h^{-1+\frac{1}{1+\delta}} d_{n}^{-\gamma}=1$. Together with the above results,

$$
\sum_{s=1}^{n-1} \operatorname{Cov}\left(R_{1}(u), R_{s+1}(u)\right)=o(1 / h)
$$

and

$$
\begin{aligned}
\operatorname{Var}\left(A_{2}(u)\right)= & \frac{V_{j}^{\top}\left[\nu_{K}^{2} \operatorname{Var}\left(\tilde{\epsilon}_{1}\right)+\frac{1}{4} \nu_{K * K}^{2}\left(\left(\varrho(u) \varrho^{\top}(u)\right) \circ \operatorname{Var}\left(\tilde{\xi}_{1}\right)\right)-\nu_{K, K * K} E\left\{\tilde{\epsilon}_{1}\left(\varrho^{\top}(u) \circ \tilde{\xi}_{1}^{\top}\right)\right\}\right] V_{j}}{n h f_{U}(u)} \\
& +o\left(\frac{1}{n h}\right) .
\end{aligned}
$$


Therefore, as $n \rightarrow \infty, h \rightarrow 0$, similar to other nonparametric estimators for strong mixing time series, the following asymptotic normality could be established,

$$
\sqrt{n h}\left(\hat{F}_{j}(u)-F_{j}(u)-\left(\frac{1}{2} w_{2}^{K} h^{2}\right) E A_{1}(u)\right) \stackrel{d}{\longrightarrow} N\left(0, \sigma_{F_{j}}^{2}\right),
$$

where

$$
\begin{aligned}
E A_{1}(u)= & {\left[F_{j}^{\prime \prime}(u)-E F_{j}^{\prime \prime}(U)\right]-\frac{1}{2} \mathrm{~V}_{j}^{\top}[(\varrho(u) \circ \sigma(u))-E(\varrho(U) \circ \sigma(U))] } \\
& +E\left[F_{i}(U) F_{j}^{\prime \prime}(U) \mathrm{V}_{i}^{\top}+F_{j}(U) F_{i}^{\prime \prime}(U) \mathrm{V}_{i}^{\top}-\frac{1}{2} \mathrm{~V}_{j}^{\top}(\varrho(U) \circ \sigma(U)) F_{i}(U) \mathrm{V}_{i}^{\top}\right. \\
& \left.-\frac{1}{2}\left(\varrho^{\top}(U) \circ \sigma^{\top}(U)\right) F_{j}(U)\right]\left(\lambda_{j}-\boldsymbol{\Lambda}\right)^{+} \mathbf{V}_{1}^{*} \mathrm{~F}(u), \\
\sigma_{F_{j}}^{2}= & \frac{V_{j}^{\top}\left[\nu_{K}^{2} \operatorname{Var}\left(\tilde{\epsilon}_{1}\right)+\frac{1}{4} \nu_{K * K}^{2}\left(\left(\varrho(u) \varrho^{\top}(u)\right) \circ \operatorname{Var}\left(\tilde{\xi}_{1}\right)\right)-\nu_{K, K * K} E\left\{\tilde{\epsilon}_{1}\left(\varrho^{\top}(u) \circ \tilde{\xi}_{1}^{\top}\right)\right\}\right] V_{j}}{f_{U}(u)} .
\end{aligned}
$$

Proof of Theorem 3.4. Let

$$
\hat{\tilde{\epsilon}}_{t}=\left(\begin{array}{c}
\hat{\epsilon}_{1,2, t} \\
\vdots \\
\hat{\epsilon}_{1, m, t} \\
\hat{\epsilon}_{2,3, t} \\
\vdots \\
\hat{\epsilon}_{2, m, t} \\
\vdots \\
\hat{\epsilon}_{m-1, m, t}
\end{array}\right), \quad \hat{\tilde{\varepsilon}}_{t}=\left(\begin{array}{c}
\hat{\varepsilon}_{1, t} \hat{\varepsilon}_{2, t} \\
\vdots \\
\hat{\varepsilon}_{1, t} \\
\hat{\varepsilon}_{2, t} \hat{\varepsilon}_{3, t} \\
\vdots \\
\hat{\varepsilon}_{2, t} \\
\vdots \\
\hat{\varepsilon}_{m, t} \\
\hat{\varepsilon}_{m-1, t} \hat{\varepsilon}_{m, t}
\end{array}\right), \quad \hat{\mathbf{B}}=\left(\hat{\mathrm{V}}_{1}, \ldots, \hat{\mathrm{V}}_{p}\right), \quad \hat{\mathrm{F}}_{[1: p]}\left(U_{t}\right)=\left(\begin{array}{c}
\hat{F}_{1}\left(U_{t}\right) \\
\vdots \\
\hat{F}_{p}\left(U_{t}\right)
\end{array}\right) .
$$

Lemma A.3. Let $\hat{p}$ be the minimizer of the information criteria defined in (2.23) among $0 \leq p \leq p_{\max }$ with $p_{\max }>p_{0}$ being a fixed search limit. Consider the cases that $p \leq p_{0}$, under the regularity conditions given before, $\hat{\sigma}_{[p]}^{2}-\frac{1}{M} E\left\|\tilde{\epsilon}_{1}\right\|^{2} \rightarrow \frac{1}{M} \operatorname{tr}\left(\mathbf{D}_{\left[p+1: p_{0}\right]}^{*}\right)$ in probability and $\operatorname{tr}\left(\mathbf{D}_{\left[p+1: p_{0}\right]}^{*}\right)=0$ for $p=p_{0}$.

Proof of Lemma A.3. For $p \leq p_{0}$,

$$
\begin{aligned}
M \hat{\sigma}_{[p]}^{2} & =\frac{1}{n} \sum_{t=1}^{n}\left\|\hat{\tilde{\varepsilon}}_{t}-\hat{a}-\hat{\mathbf{B}} \hat{\mathrm{F}}\left(U_{t}\right)\right\|^{2}=\frac{1}{n} \sum_{t=1}^{n}\left\|\hat{\tilde{\varepsilon}}_{t}-\hat{a}-\hat{\mathbf{V}}_{1,[p]}^{*} \hat{\mathrm{F}}_{[1: p]}\left(U_{t}\right)\right\|^{2} \\
& =\frac{1}{n} \sum_{t=1}^{n}\left\|\hat{\tilde{\varepsilon}}_{t}-\hat{a}-\hat{\mathbf{V}}_{1,[p]}^{*} \hat{\mathbf{V}}_{1,[p]}^{* \top} \hat{\mathrm{G}}\left(U_{t}\right)\right\|^{2} \\
& =\frac{1}{n} \sum_{t=1}^{n}\left\|\hat{\tilde{\varepsilon}}_{t}-\hat{\varrho}\left(U_{t}\right)+\left(\mathbf{I}-\hat{\mathbf{V}}_{1,[p]}^{*} \hat{\mathbf{V}}_{1,[p]}^{* \top}\right)\left(\hat{\varrho}\left(U_{t}\right)-\hat{a}\right)\right\|^{2} .
\end{aligned}
$$


Define $M \sigma_{[p]}^{* 2}=\frac{1}{n} \sum_{t=1}^{n}\left\|\tilde{\varepsilon}_{t}-a-\mathbf{V}_{1,[p]}^{*} \mathbf{V}_{1,[p]}^{* \top} \mathrm{G}\left(U_{t}\right)\right\|^{2}$, recall that $\varrho\left(U_{t}\right)=a+\mathrm{G}\left(U_{t}\right), \mathrm{F}\left(U_{t}\right)=$ $\mathrm{F}_{[1: p]}\left(U_{t}\right)=\mathrm{V}_{1,[p]}^{* \top} \mathrm{G}\left(U_{t}\right), \mathbf{B}=\mathbf{V}_{1,[p]}^{*}$, and $\mathbf{V}_{1,[p]}^{* \top} \mathbf{V}_{1,[p]}^{*}=\mathbf{I}_{p}, \mathbf{V}_{1,[p]}^{* \top} \mathbf{V}_{1,\left[p+1: p_{0}\right]}^{*}=0$, thus

$$
\begin{aligned}
M \sigma_{[p]}^{* 2} & =\frac{1}{n} \sum_{t=1}^{n}\left\|\tilde{\varepsilon}_{t}-a-\mathbf{V}_{1,[p]}^{*} \mathbf{V}_{1,[p]}^{* \top} \mathrm{G}\left(U_{t}\right)\right\|^{2} \\
& =\frac{1}{n} \sum_{t=1}^{n}\left\|\tilde{\varepsilon}_{t}-\varrho\left(U_{t}\right)+\varrho\left(U_{t}\right)-a-\mathbf{V}_{1,[p]}^{*} \mathbf{V}_{1,[p]}^{* \top} \mathrm{G}\left(U_{t}\right)\right\|^{2} \\
& =\frac{1}{n} \sum_{t=1}^{n}\left\|\tilde{\varepsilon}_{t}-\varrho\left(U_{t}\right)+\left(\mathbf{I}-\mathbf{V}_{1,[p]}^{*} \mathbf{V}_{1,[p]}^{* \top}\right)\left(\mathbf{V}_{1,[p]}^{*} \mathrm{~F}_{[1: p]}\left(U_{t}\right)+\mathbf{V}_{1,\left[p+1: p_{0}\right]}^{*} \mathrm{~F}_{\left[p+1: p_{0}\right]}\left(U_{t}\right)\right)\right\|^{2} \\
& \left.=\frac{1}{n} \sum_{t=1}^{n} \| \tilde{\epsilon}_{t}+\mathbf{V}_{1,\left[p+1: p_{0}\right]}^{*} \mathrm{~F}_{\left[p+1: p_{0}\right]}\left(U_{t}\right)\right) \|^{2} \\
& =\frac{1}{n} \sum_{t=1}^{n}\left[\tilde{\epsilon}_{t}^{\top} \tilde{\epsilon}_{t}+2 \mathrm{~F}_{\left[p+1: p_{0}\right]}^{\top}\left(U_{t}\right) \mathbf{V}_{1,\left[p+1: p_{0}\right]}^{* \top} \tilde{\epsilon}_{t}+\mathrm{F}_{\left[p+1: p_{0}\right]}^{\top}\left(U_{t}\right) \mathrm{F}_{\left[p+1: p_{0}\right]}\left(U_{t}\right)\right] .
\end{aligned}
$$

Therefore, by law of large numbers,

$$
M \sigma_{[p]}^{* 2} \rightarrow E \tilde{\epsilon}_{1}^{\top} \tilde{\epsilon}_{1}+E\left(\mathrm{~F}_{\left[p+1: p_{0}\right]}^{\top}\left(U_{1}\right) \mathrm{F}_{\left[p+1: p_{0}\right]}\left(U_{1}\right)\right)=E \tilde{\epsilon}_{1}^{\top} \tilde{\epsilon}_{1}+\operatorname{tr}\left(\mathbf{D}_{\left[p+1: p_{0}\right]}^{*}\right) \text { a.s. }
$$

Furthermore,

$$
\begin{aligned}
& M\left(\hat{\sigma}_{[p]}^{2}-\sigma_{[p]}^{* 2}\right) \\
& =\frac{1}{n} \sum_{t=1}^{n}\left[\left(\hat{\tilde{\varepsilon}}_{t}-\hat{\varrho}\left(U_{t}\right)\right)^{\top}\left(\hat{\tilde{\varepsilon}}_{t}-\hat{\varrho}\left(U_{t}\right)\right)+2 \hat{\mathrm{G}}^{\top}\left(U_{t}\right)\left(\mathbf{I}-\hat{\mathbf{V}}_{1,[p]}^{*} \hat{\mathbf{V}}_{1,[p]}^{* \top}\right)\left(\hat{\tilde{\epsilon}}_{t}-\hat{\varrho}\left(U_{t}\right)\right)\right. \\
& \left.+\hat{\mathrm{G}}^{\top}\left(U_{t}\right)\left(\mathbf{I}-\hat{\mathbf{V}}_{1,[p]}^{*} \hat{\mathbf{V}}_{1,[p]}^{* \top}\right) \hat{\mathrm{G}}\left(U_{t}\right)\right]-\frac{1}{n} \sum_{t=1}^{n}\left[\tilde{\epsilon}_{t}^{\top} \tilde{\epsilon}_{t}+2 \mathrm{~F}_{\left[p+1: p_{0}\right]}^{\top}\left(U_{t}\right) \mathbf{V}_{1,\left[p+1: p_{0}\right]}^{* \top} \tilde{\epsilon}_{t}\right. \\
& \left.+\mathrm{F}_{\left[p+1: p_{0}\right]}^{\top}\left(U_{t}\right) \mathrm{F}_{\left[p+1: p_{0}\right]}\left(U_{t}\right)\right] \\
& =\frac{1}{n} \sum_{t=1}^{n}\left[\left(\hat{\tilde{\varepsilon}}_{t}-\hat{\varrho}\left(U_{t}\right)\right)^{\top}\left(\hat{\tilde{\varepsilon}}_{t}-\hat{\varrho}\left(U_{t}\right)\right)-\tilde{\epsilon}_{t}^{\top} \tilde{\epsilon}_{t}+2 \hat{G}^{\top}\left(U_{t}\right)\left(\mathbf{I}-\hat{\mathbf{V}}_{1,[p]}^{*} \hat{\mathbf{V}}_{1,[p]}^{* \top}\right)\left(\hat{\tilde{\epsilon}}_{t}-\hat{\varrho}\left(U_{t}\right)\right)\right. \\
& \left.-2 \mathrm{~F}_{\left[p+1: p_{0}\right]}^{\top}\left(U_{t}\right) \mathbf{V}_{1,\left[p+1: p_{0}\right]}^{* \top} \tilde{\epsilon}_{t}+\hat{\mathrm{G}}^{\top}\left(U_{t}\right)\left(\mathbf{I}-\hat{\mathbf{V}}_{1,[p]}^{*} \hat{\mathbf{V}}_{1,[p]}^{* \top}\right) \hat{\mathrm{G}}\left(U_{t}\right)-\mathrm{F}_{\left[p+1: p_{0}\right]}^{\top}\left(U_{t}\right) \mathrm{F}_{\left[p+1: p_{0}\right]}\left(U_{t}\right)\right] \\
& =O_{p}\left(h^{2}+\left(\frac{\log n}{n h}\right)^{\frac{1}{2}}\right) \text {, }
\end{aligned}
$$

by the convergence results of $\hat{\tilde{\varepsilon}}_{t}, \hat{\varrho}\left(U_{t}\right), \hat{\mathrm{G}}\left(U_{t}\right)$ and $\hat{\mathbf{V}}_{1,[p]}^{*}$, it means that

$$
M\left(\hat{\sigma}_{[p]}^{2}-\sigma_{[p]}^{* 2}\right) \rightarrow 0 \text { in probability for } p \leq p_{0}
$$

Hence, we could deduce that $\hat{\sigma}_{[p]}^{2}-\frac{1}{M} E\left\|\tilde{\epsilon}_{1}\right\|^{2} \rightarrow \frac{1}{M} \operatorname{tr}\left(\mathbf{D}_{\left[p+1: p_{0}\right]}^{*}\right)$ in probability. 
Lemma A.4. For $p>p_{0}$, under the same regularity conditions, $\hat{\sigma}_{[p]}^{2}-\hat{\sigma}_{\left[p_{0}\right]}^{2}=O_{p}\left(h^{2}+\right.$ $\left.\left(\frac{\log n}{n h}\right)^{\frac{1}{2}}\right)$.

Proof of Lemma A.4. For $p>p_{0}$,

$$
\begin{aligned}
M \hat{\sigma}_{[p]}^{2}= & \frac{1}{n} \sum_{t=1}^{n}\left\|\hat{\tilde{\varepsilon}}_{t}-\hat{a}-\hat{\mathbf{B}} \hat{\mathrm{F}}\left(U_{t}\right)\right\|^{2}=\frac{1}{n} \sum_{t=1}^{n}\left\|\hat{\tilde{\varepsilon}}_{t}-\hat{a}-\hat{\mathbf{V}}_{1,[p]}^{*} \hat{\mathrm{F}}_{[1: p]}\left(U_{t}\right)\right\|^{2} \\
= & \frac{1}{n} \sum_{t=1}^{n}\left\|\hat{\tilde{\varepsilon}}_{t}-\hat{\varrho}\left(U_{t}\right)+\left(\mathbf{I}-\hat{\mathbf{V}}_{1,\left[p_{0}\right]}^{*} \hat{\mathbf{V}}_{1,\left[p_{0}\right]}^{* \top}-\hat{\mathbf{V}}_{1,\left[p_{0}+1: p\right]}^{*} \hat{\mathbf{V}}_{1,\left[p_{0}: p\right]}^{* \top}\right)\left(\hat{\varrho}\left(U_{t}\right)-\hat{a}\right)\right\|^{2} \\
= & \frac{1}{n} \sum_{t=1}^{n}\left[\left(\hat{\tilde{\epsilon}}_{t}-\hat{\varrho}\left(U_{t}\right)\right)^{\top}\left(\hat{\tilde{\epsilon}}_{t}-\hat{\varrho}\left(U_{t}\right)\right)+2 \hat{\mathrm{G}}^{\top}\left(U_{t}\right)\left(\mathbf{I}-\hat{\mathbf{V}}_{1,\left[p_{0}\right]}^{*} \hat{\mathbf{V}}_{1,\left[p_{0}\right]}^{* \top}-\hat{\mathbf{V}}_{1,\left[p_{0}+1: p\right]}^{*}\right]\right. \\
& \left.\hat{\mathbf{V}}_{1,\left[p_{0}+1: p\right]}^{* \top}\left(\hat{\tilde{\epsilon}}_{t}-\hat{\varrho}\left(U_{t}\right)\right)+\hat{\mathrm{G}}^{\top}\left(U_{t}\right)\left(\mathbf{I}-\hat{\mathbf{V}}_{1,\left[p_{0}\right]}^{*} \hat{\mathbf{V}}_{1,\left[p_{0}\right]}^{* \top}-\hat{\mathbf{V}}_{1,\left[p_{0}+1: p\right]}^{*} \hat{\mathbf{V}}_{1,\left[p_{0}+1: p\right]}^{* \top}\right) \hat{\mathrm{G}}\left(U_{t}\right)\right], \\
M & \hat{\sigma}_{\left[p_{0}\right]}^{2}=\frac{1}{n} \sum_{t=1}^{n}\left[\left(\hat{\tilde{\epsilon}}_{t}-\hat{\varrho}\left(U_{t}\right)\right)^{\top}\left(\hat{\tilde{\epsilon}}_{t}-\hat{\varrho}\left(U_{t}\right)\right)+2 \hat{\mathrm{G}}^{\top}\left(U_{t}\right)\left(\mathbf{I}-\hat{\mathbf{V}}_{1,\left[p_{0}\right]}^{*} \hat{\mathbf{V}}_{1,\left[p_{0}\right]}^{* \top}\right)\left(\hat{\tilde{\epsilon}}_{t}-\hat{\varrho}\left(U_{t}\right)\right)\right. \\
& \left.+\hat{\mathrm{G}}^{\top}\left(U_{t}\right)\left(\mathbf{I}-\hat{\mathbf{V}}_{1,\left[p_{0}\right]}^{*} \hat{\mathbf{V}}_{1,\left[p_{0}\right]}^{* \top}\right) \hat{\mathrm{G}}\left(U_{t}\right)\right],
\end{aligned}
$$

together with $\mathrm{G}\left(U_{t}\right)=\mathbf{V}_{1,\left[p_{0}\right]}^{*} \mathrm{~F}\left(U_{t}\right), \mathbf{V}_{1,\left[p_{0}\right]}^{* \top} \mathbf{V}_{1,\left[p_{0}+1: p\right]}^{*}=\mathbf{0}$, then

$$
\begin{aligned}
M\left|\hat{\sigma}_{[p]}^{2}-\hat{\sigma}_{\left[p_{0}\right]}^{2}\right|= & \left.\mid \frac{1}{n} \sum_{t=1}^{n} 2 \hat{\mathrm{G}}^{\top}\left(U_{t}\right) \hat{\mathbf{V}}_{1,\left[p_{0}+1: p\right]}^{*} \hat{\mathbf{V}}_{1,\left[p_{0}+1: p\right]}^{* \top}\right)\left(\hat{\tilde{\epsilon}}_{t}-\hat{\varrho}\left(U_{t}\right)\right) \\
& +\frac{1}{n} \sum_{t=1}^{n} \hat{\mathrm{G}}^{\top}\left(U_{t}\right) \hat{\mathbf{V}}_{1,\left[p_{0}+1: p\right]}^{*} \hat{\mathbf{V}}_{1,\left[p_{0}+1: p\right]}^{* \top} \hat{\mathrm{G}}\left(U_{t}\right) \mid \\
= & \left.\mid \frac{1}{n} \sum_{t=1}^{n} 2 \mathrm{G}^{\top}\left(U_{t}\right) \mathbf{V}_{1,\left[p_{0}+1: p\right]}^{*} \mathbf{V}_{1,\left[p_{0}+1: p\right]}^{* \top}\right)\left(\tilde{\epsilon}_{t}-\varrho\left(U_{t}\right)\right) \\
& +\frac{1}{n} \sum_{t=1}^{n} \mathrm{G}^{\top}\left(U_{t}\right) \mathbf{V}_{1,\left[p_{0}+1: p\right]}^{*} \mathbf{V}_{1,\left[p_{0}+1: p\right]}^{* \top} \mathrm{G}\left(U_{t}\right)+O_{p}\left(h^{2}+\left(\frac{\log n}{n h}\right)^{\frac{1}{2}}\right) \mid \\
= & O_{p}\left(h^{2}+\left(\frac{\log n}{n h}\right)^{\frac{1}{2}}\right) .
\end{aligned}
$$

Now we only discuss the consistency of $I C(p)$.

For $p<p_{0}$, by Lemma A.3 and $g_{n} \stackrel{P}{\longrightarrow} 0$,

$$
I C(p)-I C\left(p_{0}\right)=\log \left(\hat{\sigma}_{[p]}^{2}\right)-\log \left(\hat{\sigma}_{\left[p_{0}\right]}^{2}\right)+\left(p-p_{0}\right) g_{n}
$$




$$
\begin{aligned}
& =\log \left(1+\frac{\hat{\sigma}_{[p]}^{2}-\hat{\sigma}_{\left[p_{0}\right]}^{2}}{\hat{\sigma}_{\left[p_{0}\right]}^{2}}\right)+\left(p-p_{0}\right) g_{n} \\
& =\left(\frac{\hat{\sigma}_{[p]}^{2}-\hat{\sigma}_{\left[p_{0}\right]}^{2}}{\hat{\sigma}_{\left[p_{0}\right]}^{2}}\right)(1+o(1))+\left(p-p_{0}\right) g_{n} \\
& \stackrel{\operatorname{Pr}\left(\mathbf{D}_{\left[p+1: p_{0}\right]}^{*}\right)}{E\left\|\tilde{\epsilon}_{1}\right\|^{2}}>0 .
\end{aligned}
$$

Then $I C(p)>I C\left(p_{0}\right)$ with probability tending to 1 .

For $p>p_{0}$, by Lemma A.4 and $g_{n} /\left(h^{2}+\left(\frac{\log n}{n h}\right)^{\frac{1}{2}}\right) \stackrel{P}{\longrightarrow} \infty$,

$$
\begin{aligned}
I C(p)-I C\left(p_{0}\right) & =\log \left(\hat{\sigma}_{[p]}^{2}\right)-\log \left(\hat{\sigma}_{\left[p_{0}\right]}^{2}\right)+\left(p-p_{0}\right) g_{n} \\
& =\left(\frac{\hat{\sigma}_{[p]}^{2}-\hat{\sigma}_{\left[p_{0}\right]}^{2}}{\hat{\sigma}_{\left[p_{0}\right]}^{2}}\right)(1+o(1))+\left(p-p_{0}\right) g_{n} \\
& =O_{p}\left(h^{2}+\left(\frac{\log n}{n h}\right)^{\frac{1}{2}}\right)+\left(p-p_{0}\right) g_{n}>0 .
\end{aligned}
$$

Therefore, $\hat{p}$ which minimizes $I C(p)$ converge to $p_{0}$ with probability going to 1 . 